\title{
Global Stability of an Economic Model with a Continuous Delay of Kaldor Type Modified
}

\author{
Aka Fulgence Nindjin, Tetchi Albin N'guessan (D), Sahoua Hypolithe Okou A Kpetihi (D), \\ and Kessé Tiban Tia
}

UFR de Mathématiques et Informatique Université Félix Houphouët Boigny d’Abidjan Cocody 22 BP 582 Abidjan 22, Côte d'Ivoire

Correspondence should be addressed to Tetchi Albin N’guessan; albintetchi@gmail.com and

Sahoua Hypolithe Okou A Kpetihi; okouakpetihi@hotmail.com

Received 7 March 2018; Revised 13 May 2018; Accepted 17 May 2018; Published 2 August 2018

Academic Editor: Dongfang Li

Copyright (C) 2018 Aka Fulgence Nindjin et al. This is an open access article distributed under the Creative Commons Attribution License, which permits unrestricted use, distribution, and reproduction in any medium, provided the original work is properly cited.

\begin{abstract}
This paper studies continuous nonlinear economic dynamics with a continuous delay of a Kaldor type modified in dimension two. The important results are, on the one hand, the boundedness of solutions, the existence of an attractive set, and the permanence of the system and, on the other hand, the local and global stability of equilibrium points.
\end{abstract}

\section{Model}

The complex nonlinear dynamic has been introduced into the analysis of economic phenomena to explain not only the fluctuations observed in the series studies but also the economic crisis in the capitalist system. Thus, economists such as Goodwin (1967) and Kaldor (1955-1956) have employed dynamic models to explain that the cyclic and chaotic growth curves are the economic phenomena endogenous to the capitalist system itself. Within the framework of structural reforms for economic dynamics, NINDJIN et al. in [1] suggest a proven model originating from ecology which could have a certain range in analysis and regulation of financial systems. This model of dimension two describes how the GDP and an economic capital interact in accordance with the model of Kaldor modified in order to increase the resilience of these dynamics against possible disruptions. Mathematical analysis of this model (see [1]) demonstrates that it is bounded and permanent and admits under certain circumstances an attractive set. On the one hand, this permanence manifests itself in the form of stationary growth of capital stock and product (stable interior equilibrium point). On the other hand, it appears in the capital cyclic growth and the product (limit cyclic). So, a capital stock rupture or a long-term production is prevented. It is also shown that the financial system stability (relative to the capital and the product) is overall; i.e., it does not depend on either stock level or production at the initial time. Facing a possible disruption of one of the control parameters of the economic system, we have analyzed, in [2], the model bifurcations. We have shown that the model admits a transcritical bifurcation, a pitchfork bifurcation or a Hopf bifurcation. In the last one, when the model is disrupted, it changes from a stationary balanced growth to a cyclic growth by preventing a GDP or capital crisis. Interactions between the GDP and the economy's capital could not be clearly and definitively understood or explained regardless of past situations which may affect the present or the future. In this paper, we are going to focus on the way in which investments are evolved and savings are established. Indeed, economies finance their investments through their own savings or those of other economies via financial structures with an interest rate. Regarding the savings, it is known that they are made up of a portion of profits or wages. Thus, when the economy is able to be selffunding by its savings, then the net profit increases $\pi_{\text {net }}=$ $\pi-r D$ (i.e., profit, $\pi$ deprived of the portion set aside to pay interest, $r$ debt, $D$ ). So, saving at a time $t$ depends on the net profit; consequently, the GDP from a time $t_{0}=t-\bar{T}$ where $\bar{T}>0$. Let us suppose that $\bar{T}$ is the deadline needed by this saving to reach a certain threshold likely to ensure the selffinancing of the investments of the economy. 
Let us consider the dynamics with no delay of the modified Kaldor type and the following assumption:

$$
\begin{aligned}
& \begin{aligned}
& \dot{Y}= {\left[a_{0} Y\left(1-\frac{Y}{C_{0}}\right)\right] } \\
&+\alpha\left(a_{1} K+b_{1}\right) Y\left(1-\frac{m_{2} K}{Y+C_{2}}\right) \\
&-\left[\alpha \frac{m_{1} Y}{Y+C_{1}}\right] K, \\
& \dot{K}= {\left[\left(a_{2} Y+b_{2}\right)\left(1-\frac{m_{2} K}{Y+C_{2}}\right)\right] K-\varrho K, } \\
& Y(0)>0, K(0)>0, Y, K \in C^{1}\left(\left[0 ;+\infty\left[; \mathbb{R}_{+}\right),\right.\right. \\
& \text {with }\left(a_{0}, C_{0}, C_{1}, C_{2}, m_{1}, m_{2}, \alpha\right) \in\left(\mathbb{R}_{+}^{*}\right)^{7} \text { and }\left(a_{1}, a_{2}, b_{1},\right.
\end{aligned} \\
& \left.b_{2}, \varrho\right) \in\left(\mathbb{R}_{+}\right)^{5} . \\
& Y \text { denotes the product, } K \text { denotes the stock of capital, and } \\
& \dot{Y} \text { and } \dot{K} \text { indicate, respectively, the growth rate of the product } \\
& \text { and the stock of capital depending on the following economic } \\
& \text { parameters: }
\end{aligned}
$$

(i) $a_{0}$ the trend of rate of increase in GDP for a given (future) period in absence (or in neglect) of the losses,

(ii) $C_{0}$ the maximum (monetary) value of GDP we can get from this economy for that given period,

(iii) $\alpha$ the currency adjustment factor,

(iv) $m_{1}$ the maximum (monetary) value of the genuine saving of this economy for the given period,

(v) $C_{1}$ the maximum (monetary) value of the saving supported by the economy in the given period,

(vi) $m_{2}$ the maximum value of the investment rates losses for the given period,

(vii) $C_{2}$ the maximum capital stock for the given period,

(viii) $a_{1}$ the derivative relative to the capital of the investment rate in the absence (or in the neglect) of the losses (when $m_{2}=0$ ) for the given period,

(ix) $b_{1}$ the investment rate when the capital is null ( $K=$ $0)$ and this rate has suffered no loss $\left(m_{2}=0\right)$ for the given period,

(x) $a_{2}$ the share of the GDP converted into stock of capital for the given period,

(xi) $b_{2}$ the accumulation rate of capital when the product is null $(Y=0)$ and that the investment rate has suffered no loss $\left(m_{2}=0\right)$ for the given period.

(see [1], page 4 ).

Assumption 1. The ratio saving-capital $g(Y, K)=m_{1} Y /(Y+$ $C_{1}$ ) at a given time $t$ depends on the GDP produced since $t_{0}=$ $t-\bar{T}$ with a probability of exponential lag (see, [3], appendix, page 272).
So, the ratio saving-capital with delay is $\tilde{g}(t, \bar{T})=$ $m_{1} x(t) /\left(x(t)+C_{1}\right)$ with $x(t)=\int_{t-\bar{T}}^{t} \mu e^{-\mu(t-\xi)} Y(\xi) d \xi$ so that $\int_{-\infty}^{t} \mu e^{-\mu(t-\xi)} d \xi=1, \mu \in \mathbb{R}_{+}^{*}$. Then,

$$
\dot{x}=-\mu(x-Y)-\mu e^{-\mu \bar{T}} Y(t-\bar{T}) .
$$

By replacing $g(Y, K)$ by $\widetilde{g}(t, \bar{T})$, one obtains continuous nonlinear economic dynamics with a continuous delay of a Kaldor type modified in dimension two. Enclosing (1) to the system with a continuous delay, one gets the following system with discrete delay in dimension three:

$$
\begin{aligned}
\dot{Y}= & a_{0}\left[1-\frac{Y}{C_{0}}\right] Y \\
& +\alpha\left(a_{1} K+b_{1}\right)\left[\left(1-\frac{m_{2} K}{Y+C_{2}}\right)\right] Y \\
& -\alpha\left[\frac{m_{1} x}{x+C_{1}}\right] K
\end{aligned}
$$

$$
\begin{aligned}
& \dot{K}=\left(a_{2} Y+b_{2}\right)\left[\left(1-\frac{m_{2} K}{Y+C_{2}}\right)\right] K-\varrho K, \\
& \dot{x}=-\mu(x-Y)-\mu e^{-\mu \bar{T}} Y(t-\bar{T}),
\end{aligned}
$$$$
Y, K, x \in C^{1}\left(\left[0 ;+\infty\left[; \mathbb{R}_{+}\right), Y(0)>0, K(0)>0,\right.\right.
$$

with $\left(a_{0}, a_{1}, a_{2}, b_{1}, b_{2}, c_{0}, c_{1}, c_{2}, m_{1}, m_{2}, \varrho, \alpha, \bar{T}, \mu\right) \in\left(\mathbb{R}_{+}^{*}\right)^{14}$, $Y_{t}(\theta)=Y(t+\theta), \forall \theta \in[-\bar{T} ; 0]$, and the feedback function $Y_{0}=\varphi_{1} \in C\left([-\bar{T} ; 0] ; \mathbb{R}_{+}\right)$is consequently $Y_{0}(0)=Y(0)$.

To facilitate the qualitative study of system (1.2) which possesses 14 parameters $\left(a_{0}, a_{1}, a_{2}, b_{1}, b_{2}, c_{0}, c_{1}, c_{2}\right.$, $\left.m_{1}, m_{2}, \varrho, \alpha, \bar{T}, \mu\right) \in\left(\mathbb{R}_{+}^{*}\right)^{14}$, let us change the variables by reducing the number of parameters.

Let us define the new variables:

$$
\begin{aligned}
\tau & =a_{0} t, \\
u(\tau) & =\frac{Y(t)}{c_{0}}, \\
v(\tau) & =\frac{m_{2}}{c_{0}} \mathrm{~K}(\mathrm{t}), \\
w(t) & =\frac{x}{c_{0}}=\int_{t-T}^{t} \lambda e^{-\lambda(t-\xi)} u(\xi) d \xi .
\end{aligned}
$$

Let us define the new parameters of control:

$$
\begin{aligned}
& \beta_{1}=\frac{\alpha a_{1} C_{0}}{a_{0} m_{2}}, \\
& \beta_{2}=\frac{a_{2} C_{0}}{a_{0}}, \\
& \alpha_{1}=\frac{\alpha b_{1}}{a_{0}},
\end{aligned}
$$




$$
\begin{aligned}
\alpha_{2} & =\frac{b_{2}}{a_{0}}, \\
d_{1} & =\frac{C_{1}}{C_{0}}, \\
d_{2} & =\frac{C_{2}}{C_{0}}, \\
\gamma & =\alpha \frac{m_{1}}{a_{0} m_{2}}, \\
\delta & =\frac{\varrho}{a_{0}},
\end{aligned}
$$

$T=a_{0} \bar{T}$, and $\lambda=\mu / a_{0}$. Then, system (1.2) becomes

$$
\begin{aligned}
\dot{u}(\tau)= & {[1-u(\tau)] u(\tau) } \\
& +\left(\beta_{1} v(\tau)+\alpha_{1}\right)\left(1-\frac{v(\tau)}{u(\tau)+d_{2}}\right) u(\tau) \\
& -\frac{\gamma w(\tau)}{w(\tau)+d_{1}} v(\tau), \\
\dot{v}(\tau)= & \left(\beta_{2} u(\tau)+\alpha_{2}\right)\left(1-\frac{v(\tau)}{u(\tau)+d_{2}}\right) v(\tau)-\delta v(\tau),
\end{aligned}
$$

$$
\begin{aligned}
& \dot{w}(\tau)=-\lambda(w(\tau)-u(\tau))-\lambda e^{-\lambda T} u(\tau-T), \\
& u, v, w \in C^{1}\left(\left[0 ;+\infty\left[; \mathbb{R}_{+}\right) u(0)>0, v(0)>0, w(0)>0,\right.\right.
\end{aligned}
$$

with $\left(d_{1}, d_{2}, \gamma, \alpha_{1}, \alpha_{2}, \beta_{1}, \beta_{2}, \delta, T, \lambda\right) \in\left(\mathbb{R}_{+}^{*}\right)^{10}$ and $u_{0}=$ $\varphi \in C\left([-T ; 0] ; \mathbb{R}_{+}\right)$so that $u_{0}(0)=u(0)$.

In the long run, we shall adopt the following notations:

$$
\begin{gathered}
\|\varphi\|=\sup _{\theta \in[-T ; 0]}\{|\varphi(\theta)|\}, \\
k=1-e^{-\lambda T}
\end{gathered}
$$

$$
\text { and } w(0)=\int_{-T}^{0} \lambda \varphi(\theta) e^{\lambda \theta} d \theta \text {. }
$$

\section{Boundedness and Equilibria Points of (1.3)}

2.1. Boundedness of the Solutions of Model (1.3)

Lemma 2. The interior $\operatorname{int}\left(\mathbb{R}_{+}^{3}\right)$ and the boundary $\partial\left(\mathbb{R}_{+}^{3}\right)$ of a positive cone $\mathbb{R}_{+}^{3}$ are invariant for model (1.3).

Proof. Given $0 \leq T_{0}<+\infty$. Let $\tau$ be in $\left[-T ; T_{0}\right.$ [. One knows that

$$
\begin{aligned}
& u(\tau)=u(0) \exp \left\{\int_{0}^{\tau}\left[1-u(t)+\left(\beta_{1} v(t)+\alpha_{1}\right)\left(1-\frac{v(t)}{u(t)+d_{2}}\right)-\frac{\gamma v(t) w(t)}{u(t)\left(w(t)+d_{1}\right)}\right] d t\right\}, \\
& v(\tau)=v(0) \exp \left\{\int_{0}^{\tau}\left[\left(\beta_{2} u(t)+\alpha_{2}\right)\left(1-\frac{v(t)}{u(t)+d_{2}}\right)-\delta\right] d t\right\}, \\
& w(\tau)=w(0) \exp \left\{\int_{0}^{\tau} \frac{-\lambda(w(t)-u(t))-\lambda e^{-\lambda T} u(t-T)}{w(t)} d t\right\} .
\end{aligned}
$$

Thus, on the one hand, $(u(0), v(0), w(0)) \in \partial\left(\mathbb{R}_{+}^{3}\right) \Longrightarrow$ $(u(\tau), v(\tau), w(\tau)) \in \partial\left(\mathbb{R}_{+}^{3}\right)$, and, on the other hand, $(u(0)$, $v(0), w(0)) \in \operatorname{int}\left(\mathbb{R}_{+}^{3}\right) \Longrightarrow(u(\tau), v(\tau), w(\tau)) \in \operatorname{int}\left(\mathbb{R}_{+}^{3}\right)$.

Lemma 3 (see [4]). Given $(A, B) \in \mathbb{R}_{+}^{2}$ and $\phi$ a continuous and derivable function as there is $t_{0} \geq 0$ verifying $\phi\left(t_{0}\right)>0$. Then, $\forall t \geq t_{0}$,

$$
\begin{aligned}
\frac{d \phi}{d t} & \leq \phi(B-A \phi) \Longrightarrow \\
\lim _{t \rightarrow+\infty} \sup _{t \rightarrow \infty}(t) & \leq \frac{B}{A}, \\
\frac{d \phi}{d t} & \geq \phi(B-A \phi) \Longrightarrow \\
\liminf _{t \rightarrow+\infty} \phi(t) & \geq \frac{B}{A} .
\end{aligned}
$$

Theorem 4. Let us assume that $\alpha_{2}>\delta$. Let us pose

$$
\begin{aligned}
& M_{u}=\frac{4 d_{2} \beta_{1}+\left(\beta_{1} d_{2}+2 \alpha_{1}\right) \beta_{1} d_{2}+\alpha_{1}^{2}}{\beta_{1} d_{2}\left(4-\beta_{1}\right)} \text { if } 0<\beta_{1}<4 \\
& \text { and } M_{u}=1+\alpha_{1} \quad \text { if } \beta_{1}=0, \\
& M_{v}=\frac{\left(M_{u}+d_{2}\right)\left(\beta_{2} M_{u}+\alpha_{2}-\delta\right)}{\alpha_{2}} \\
& \text { and } m_{v}=\frac{\left(\alpha_{2}-\delta\right) d_{2}}{\beta_{2} d_{2}+\alpha_{2}} \\
& m_{u}^{(0)}=m^{u}+\beta_{1} m_{v}, \\
& m^{u}=-\frac{\beta_{1}}{d_{2}} M_{v}^{2}-\frac{\alpha_{1}}{d_{2}} M_{v}+1+\alpha_{1}, \\
& m_{u}^{\varepsilon}=\frac{m_{u}^{(0)}+\sqrt{\left[m_{u}^{(0)}-\varepsilon\right]^{2}-4 \gamma k M_{u} M_{v} / d_{1}}}{2}
\end{aligned}
$$




$$
\begin{aligned}
& \quad 0 \leq \varepsilon \leq m_{u}^{(0)}-2 \sqrt{\frac{\gamma k M_{u} M_{v}}{d_{1}}} \\
& \text { or } m_{u}^{(0)}+2 \sqrt{\frac{\gamma k M_{u} M_{v}}{d_{1}}} \leq \varepsilon \\
& M_{w}=k M_{u} \\
& \text { and } m_{w}=k m_{u}^{\varepsilon} . \\
& \text { If } m_{u}^{(0)} \geq 2 \sqrt{\frac{\gamma k M_{u} M_{v}}{d_{1}}}
\end{aligned}
$$

and, then, model (1.3) is permanent. Otherwise, the set defined by

$$
\begin{aligned}
\mathscr{A}^{+} & =\left\{(u, v, w) \in \mathbb{R}_{+}^{3}: m_{u}^{\varepsilon} \leq u \leq M_{u}, m_{v} \leq v\right. \\
& \left.\leq M_{v} \text { and } m_{w} \leq w \leq M_{w}\right\}
\end{aligned}
$$

Which is a bounded set, positively invariant for model (1.3).

Proof. Let us consider system (1.3) and Lemma 3.

(1) One has the following: $\dot{v}(\tau)$ does not depend on the variable $w$. So, one obtains the same results like those of the model with no delay, i.e.,

$\liminf _{t \longrightarrow+\infty}[v(t)] \geq m_{v}$ and $\lim \sup _{t \rightarrow+\infty}[v(t)] \leq$ $M_{v}$.

(2) One has the following: $d u / d t \leq(1-u) u+\left(\beta_{1} v+\alpha_{1}\right)(1-$ $\left.v /\left(u+d_{2}\right)\right) u$ because $-\gamma w v /\left(w+d_{1}\right)<0$.

Then, $d u / d t \leq\left(B_{1}-A_{1} u\right) u$ with $\left(A_{1}, B_{1}\right)=\left(1 ; 1+\alpha_{1}\right)$ if $\beta_{1}=0$

and $\left(A_{1}, B_{1}\right)=\left(\left(4-\beta_{1}\right) / 4 ;\left(4 d_{2} \beta_{1}+\left(\beta_{1} d_{2}+2 \alpha_{1}\right) \beta_{1} d_{2}+\right.\right.$ $\left.\left.\alpha_{1}^{2}\right) / 4 \beta_{1} d_{2}\right)$ if $0<\beta_{1}<4$ and, in that case, $\max _{v}\left\{\left(\beta_{1} v+\right.\right.$ $\left.\left.\alpha_{1}\right)\left(1-v /\left(u+d_{2}\right)\right) u\right\}=u\left[\beta_{1}\left(u+d_{2}\right)+\alpha_{1}\right]^{2} / 4 \beta_{1}\left(u+d_{2}\right)$.

By property (6) of Lemma 3, one obtains $\lim \sup _{t \rightarrow+\infty}[u(t)] \leq M_{u}$.

(3) One knows that $0 \leq u(\tau)$. Let us suppose that $u(0)>0$ and, then, there exist $t_{0}$ so that, $\forall \tau \geq t_{0}, 0<u(\tau) \leq$ $M_{u}$. Let us suppose that $l=\liminf _{t \rightarrow+\infty}[u(t)]$. Thus, for any $\varepsilon$ very tiny, there is $t_{1}>0$ so that, $\forall \tau>t_{1}$, $u(\tau) \geq l-\varepsilon>0$.
Let us pose $t_{2}=\max \left(t_{0}, t_{1}\right)$ and $m=l-\varepsilon$. Then, $\forall \tau>$ $t_{2}, 0<m<u(\tau) \leq M_{u}$. Thus

$$
\begin{aligned}
& \forall \tau>t_{2}, \\
& 0<w(\tau)<\frac{u(\tau) w(\tau)}{m} \leq \frac{M_{u} w(\tau)}{m}
\end{aligned}
$$

Hence, $\forall \tau>t_{2},-\gamma w(\tau) /\left(w(\tau)+d_{1}\right)>$ $-\gamma(u(\tau) w(\tau) / m) /\left(u(\tau) w(\tau) / m+d_{1}\right)$ for $g(x)=$ $-\gamma x /\left(x+d_{1}\right)$ is decreasing upon $\mathbb{R}_{+}$. On the one hand,

it is known that $w(t)=\int_{t-T}^{t} \lambda e^{-\lambda(t-\xi)} u(\xi) d \xi$ and $\int_{-\infty}^{t} \lambda e^{-\lambda(t-\xi)} d \xi=1$.

Meanwhile, $\lim \sup _{t \rightarrow+\infty}\left\{\int_{t-T}^{t} \lambda e^{-\lambda(t-\xi)} u(\xi) d \xi\right\} \leq$ $\int_{t-T}^{t} \lambda e^{-\lambda(t-\xi)} d \xi \times \lim \sup _{t \rightarrow+\infty}[u(t)]$ and, then,

$\lim \sup _{\tau \longrightarrow+\infty}[w(\tau)] \leq M_{u}\left(1-e^{-\lambda T}\right)=k M_{u}=M_{w}$. On the other hand,

$\dot{u} / u=[1-u(\tau)]+\left(\beta_{1} v(\tau)+\alpha_{1}\right)(1-v(\tau) /(u(\tau)+$ $\left.\left.d_{2}\right)\right)-\left(\gamma w(\tau) /\left(w(\tau)+d_{1}\right)\right)(v(\tau) / u(\tau))$ and $1+\left(\beta_{1} v(\tau)+\right.$ $\left.\alpha_{1}\right)\left(1-v(\tau) /\left(u(\tau)+d_{2}\right)\right) \geq m_{u}^{(0)}=-\left(\beta_{1} / d_{2}\right) M_{v}^{2}-$ $\left(\alpha_{1} / d_{2}\right) M_{v}+1+\alpha_{1}+\beta_{1} m_{v}$. Then, $\tau>t_{2}$, and $\dot{u} \geq\left[m_{u}^{(0)}-\gamma w(\tau) v(\tau) /\left(u(\tau) w(\tau)+m d_{1}\right)-u\right] u(\tau)$. So, $d u / d t \geq\left(B_{2}-A_{2} u\right) u$ with $A_{2}=1$ and $B_{2}=$ $m_{u}^{(0)}-\gamma M_{v} M_{w} / m d_{1}$. Therefore, applying property (7) of Lemma 3 one obtains $\liminf _{t \rightarrow+\infty}[u(t)] \geq m_{u}^{(0)}-$ $\gamma M_{v} M_{w} / m d_{1}$.

Consequently, $l \geq m_{u}^{(0)}-\gamma M_{v} M_{w} /(l-\varepsilon) d_{1}$ for $m=l-\varepsilon$. So, one obtains

$$
l^{2}-\left(m_{u}^{(0)}+\varepsilon\right) l+\frac{\gamma M_{u} M_{w}}{d_{1}}+m_{u}^{(0)} \varepsilon \geq 0 .
$$

If $m_{u}^{(0)} \geq 2 \sqrt{\gamma k M_{u} M_{v} / d_{1}}$ then $\left[m_{u}^{(0)}-\varepsilon\right]^{2}-$ $4 \gamma M_{w} M_{v} / d_{1} \geq 0$.

Thus one obtains $\liminf _{t \rightarrow+\infty}[u(t)] \geq m_{u}^{\varepsilon}=\left(m_{u}^{(0)}+\right.$ $\sqrt{\left.\left[m_{u}^{(0)}-\varepsilon\right]^{2}-4 \gamma M_{w} M_{v} / d_{1}\right)} / 2$

with $0 \leq \varepsilon \leq m_{u}^{(0)}-2 \sqrt{\gamma k M_{u} M_{v} / d_{1}}$ or $m_{u}^{(0)}+$ $2 \sqrt{\gamma k M_{u} M_{v} / d_{1}} \leq \varepsilon$.

(4) It is known that $\lim \inf _{t \rightarrow+\infty}\left\{\int_{t-T}^{t} \lambda e^{-\lambda(t-\xi)} u(\xi) d \xi\right\} \geq$ $\int_{t-T}^{t} \lambda e^{-\lambda(t-\xi)} d \xi \quad \times \quad \liminf _{t \rightarrow+\infty}[u(t)] . \quad$ So, $\liminf _{t \rightarrow+\infty}[w(t)] \geq m_{w}=k m_{u}^{\varepsilon}$.

Remark 5. Let us consider the notations of Theorem 4. 
(1) Let us consider the following assumptions:

$$
\begin{aligned}
\alpha_{2} & >\delta, \\
m_{u}^{(0)} & >0, \\
\frac{d_{1}\left[m_{u}^{(0)}\right]^{2}}{4 \gamma M_{u} M_{v}} & <1 \\
\text { and } T & <-\frac{1}{\lambda} \ln \left[1-\frac{d_{1}\left[m_{u}^{(0)}\right]^{2}}{4 \gamma M_{u} M_{v}}\right] . \\
\alpha_{2} & >\delta, \\
m_{u}^{(0)} & >0 \\
\text { and } 0 & \leq \gamma<\frac{d_{1}\left[m_{u}^{(0)}\right]^{2}}{4 k M_{u} M_{v}} .
\end{aligned}
$$

One remarks that each of assumptions (13) and (14) implies, respectively, condition (9).

(2) If $m_{u}^{(0)} \geq 2 \sqrt{\gamma k M_{u} M_{v} / d_{1}}$, one has $0<m_{u}^{(0)} / 2 \leq m_{u}^{\varepsilon} \leq$ $\lim \inf _{t \rightarrow+\infty}[u(t)]$. So, we can adjust the minimum value of $\lim \inf _{t \rightarrow+\infty}[u(t)]$ by choosing the value of $\varepsilon$.

\subsection{The Equilibria Points of Model (1.3)}

2.2.1. Points of Trivial Equilibria. If $0<\alpha_{2} \leq \delta$ then system (1.3) admits two points of trivial equilibria: $E_{0}^{*}=$ $(0 ; 0 ; 0), E_{1}^{*}=\left(1+\alpha_{1} ; 0 ; k\left(1+\alpha_{1}\right)\right)$.

If $\alpha_{2}>\delta \geq 0$ then system (1.3) admits three points of trivial equilibria: $E_{0}^{*}=(0 ; 0 ; 0), E_{1}^{*}=\left(1+\alpha_{1} ; 0 ; k\left(1+\alpha_{1}\right)\right)$, and $E_{2}^{*}=\left(0 ;\left(\alpha_{2}-\delta\right) d_{2} / \alpha_{2} ; 0\right)$
2.2.2. Points of Interior Equilibria. Given $p_{1}(x)=a_{14} x^{4}+$ $a_{13} x^{3}+a_{12} x^{2}+a_{11} x+a_{10}$ such as

$$
\begin{aligned}
a_{14}= & -k \beta_{2}^{2}, \\
a_{13}= & \left(k-d_{1}\right) \beta_{2}^{2}-2 k \beta_{2} \alpha_{2}+k \beta_{2}\left(\beta_{1} \delta-\gamma \beta_{2}\right), \\
a_{12}= & 2 \beta_{2} \alpha_{2}\left(k-d_{1}\right)-k \alpha_{2}^{2}+d_{1} \beta_{2}^{2}+k \alpha_{1} \delta \beta_{2} \\
& -k \gamma \beta_{2} \alpha_{2}+\beta_{1} \beta_{2} \delta d_{1} \\
& +k\left(\beta_{2} d_{2}+\alpha_{2}-\delta\right)\left(\beta_{1} \delta-\gamma \beta_{2}\right), \\
a_{11}= & \alpha_{1} \delta\left(\beta_{2} d_{1}+k \alpha_{2}\right)+\left(k-d_{1}\right) \alpha_{2}^{2}+2 \beta_{2} \alpha_{2} d_{1} \\
& +k\left(\beta_{1} \delta-\gamma \beta_{2}\right) d_{2}\left(\alpha_{2}-\delta\right) \\
& +\left(\beta_{1} \delta d_{1}-k \gamma \alpha_{2}\right)\left(\beta_{2} d_{2}+\alpha_{2}-\delta\right), \\
a_{10}= & d_{1} \alpha_{2}^{2}+\alpha_{1} \alpha_{2} \delta d_{1}+\left(\beta_{1} \delta d_{1}-k \gamma \alpha_{2}\right) d_{2}\left(\alpha_{2}-\delta\right) .
\end{aligned}
$$

(1) System (1.3) does not admit any point of interior equilibria if $\beta_{2} M_{u}+\alpha_{2}-\delta<0$.

(2) Any interior equilibrium point $E_{3}^{*}=\left(u^{*} ; v^{*}, w^{*}\right)$ of system (1.3) verifies the following relations:

$$
\begin{aligned}
p_{1}\left(u^{*}\right) & =0 \\
v^{*} & =\frac{\left(\beta_{2} u^{*}+\alpha_{2}-\delta\right)}{\beta_{2} u^{*}+\alpha_{2}}\left(u^{*}+d_{2}\right)
\end{aligned}
$$

with $\beta_{2} u^{*}+\alpha_{2}-\delta>0$,

$$
w^{*}=k u^{*} \text {. }
$$

Designating $P_{x y}$ the projection over the plan $x y$, we study the border dynamics of the model.

\section{Border Dynamics on the Plan $u v$}

On $u v$ plan, model (1.3) becomes

$$
\begin{aligned}
& \dot{u}(\tau)=[1-u(\tau)] u(\tau)+\left(\beta_{1} v(\tau)+\alpha_{1}\right)\left(1-\frac{v(\tau)}{u(\tau)+d_{2}}\right) u(\tau), \\
& \dot{v}(\tau)=\left(\beta_{2} u(\tau)+\alpha_{2}\right)\left(1-\frac{v(\tau)}{u(\tau)+d_{2}}\right) v(\tau)-\delta v,
\end{aligned}
$$

$$
u, v, w \in C^{1}\left(\left[0 ;+\infty\left[; \mathbb{R}_{+}\right), u(0)>0, v(0)>0, u_{0}=\varphi \in C\left([-T ; 0] ; \mathbb{R}_{+}\right),\right.\right.
$$

with $\left(d_{2}, \alpha_{1}, \alpha_{2}, \beta_{1}, \beta_{2}, \delta, T, \lambda\right) \in\left(\mathbb{R}_{+}^{*}\right)^{7}$.

The equilibria points of model (3.1) are

$$
\begin{aligned}
& U_{0}^{(1)}=P_{u v}\left(E_{0}^{*}\right)=(0,0), \\
& U_{1}^{(1)}=P_{u v}\left(E_{1}^{*}\right)=\left(1+\alpha_{1} ; 0\right),
\end{aligned}
$$

$$
\begin{aligned}
& U_{2}^{(1)}= P_{u v}\left(E_{2}^{*}\right)=\left(0 ; \frac{\left(\alpha_{2}-\delta\right) d_{2}}{\alpha_{2}}\right), \\
& U_{3}^{(1)}=\left(\bar{u} ; \frac{\left(\beta_{2} \bar{u}+\alpha_{2}-\delta\right)\left(\bar{u}+d_{2}\right)}{\beta_{2} \bar{u}+\alpha_{2}}\right) \\
& \quad \text { if } \bar{u}>0 \text { and }\left(\beta_{2} \bar{u}+\alpha_{2}-\delta\right)>0,
\end{aligned}
$$


where $p_{0}(\bar{u})=a_{03} \bar{u}^{3}+a_{02} \bar{u}^{2}+a_{01} \bar{u}+a_{00}=0$.

With $a_{03}=-\beta_{2}^{2}$,

$$
\begin{aligned}
a_{02}= & \left(\beta_{2}-2 \alpha_{2}+\delta \beta_{1}\right) \beta_{2}, \\
a_{01}= & \alpha_{2}\left(2 \beta_{2}-\alpha_{2}\right)+\beta_{1} \delta\left(\beta_{2} d_{2}+\alpha_{2}-\delta\right) \\
& +\delta \beta_{2} \alpha_{1}, \\
a_{00}= & \alpha_{2}^{2}+\delta \beta_{1} d_{2}\left(\alpha_{2}-\delta\right)+\alpha_{1} \alpha_{2} \delta .
\end{aligned}
$$

Let us give below the results on the permanence of the model. Therefore, let us consider the notations of Theorem 4 . If $\alpha_{2}>$ $\delta$ and $m_{u}^{(0)}>0$ then model (3.1) is permanent. Among others, the set defined by

$$
\begin{aligned}
& \mathscr{A}^{(0)} \\
& =\left\{(u, v) \in \mathbb{R}_{+}^{2}: m_{u}^{(0)} \leq u \leq M_{u} \text {, and } m_{v} \leq v \leq M_{v}\right\}
\end{aligned}
$$

which is a bounded set, positively invariant for model (3.1).

3.1. Local Stability of the Equilibria Points of Model (3.1). Performing the spectral study of Jacobian matrix of the system linearized around each of the points of equilibrium, one obtains, classically, the following conclusions:

(1) Stability of $U_{0}^{(1)}=(0 ; 0)$ :

(a) $U_{0}^{(1)}$ is an unstable node if $\alpha_{2}>\delta$.

(b) $U_{0}^{(1)}$ is a point unstable saddle repulsive following direction $u$ and attractive following direction $v$ if $\alpha_{2}<\delta$.

(2) Stability $U_{1}^{(1)}=\left(1+\alpha_{1} ; 0\right)$ :

(a) $U_{1}^{(1)}$ is stable if $\alpha_{2}+\left(1+\alpha_{1}\right) \beta_{2}<\delta$.

(b) $U_{1}^{(1)}$ is a point unstable saddle attractive following direction $u$ and repulsive following direction $w_{1}$ if $\alpha_{2}+\left(1+\alpha_{1}\right) \beta_{2}>\delta$.

(3) Stability of $U_{2}^{(1)}=\left(0 ;\left(\alpha_{2}-\delta\right) d_{2} / \alpha_{2}\right)$ for $\alpha_{2}>\delta$ :

(a) $U_{2}^{(1)}$ is stable if $\psi(\delta)<0$.

(b) $U_{2}^{(1)}$ is a point of unstable saddle if $\psi(\delta)>0$, repulsive following $w_{2}$, and attractive following v. Consider

$$
\psi(x)=-d_{2} \beta_{1} x^{2}+\left[d_{2} \beta_{1} \alpha_{2}+\alpha_{1} \alpha_{2}\right] x+\alpha_{2}^{2} .
$$

(4) Given $U_{3}^{(1)}=(\bar{u} ; \bar{v})$ interior equilibrium point of (3.1) testifying the system (19)-(20) and J its associated Jacobian matrix.

(a) $U_{3}^{(1)}$ is stable (stable node or stable focus) if $\operatorname{det}(J)>0$ and $\operatorname{Tr}(J)<0$. (b) $U_{3}^{(1)}$ is marginal or center if $\operatorname{det}(J) \geq 0$ and $\operatorname{Tr}(J)=0$.

(c) $U_{3}^{(1)}$ is unstable if $\operatorname{det}(J)<0$ or $(\operatorname{det}(J)>0$ and $\operatorname{Tr}(J)>0)$, precisely:

(i) $U_{3}^{(1)}$ is a node or a focus if $\operatorname{det}(J)>0$ and $\operatorname{Tr}(J)>0$,

(ii) $U_{3}^{(1)}$ is a point unstable saddle if $\operatorname{det}(J)<0$.

With $\operatorname{Tr}(J)$ the trace and $\operatorname{det}(J)$ determinant of J, vectors

$$
\begin{aligned}
w_{1} & =\left(\left(1+\alpha_{1}\right)\left[\beta_{1}-\frac{\alpha_{1}}{1+\alpha_{1}+d_{2}}\right] ;\left(1+\alpha_{1}\right)\left(\beta_{2}+1\right)\right. \\
& \left.+\alpha_{2}-\delta\right) .
\end{aligned}
$$

and $w_{2}=\left(\psi(\delta) / \alpha_{2}^{2}+\alpha_{2}-\delta ;\left[\beta_{2} d_{2} \delta+\alpha_{2}\left(\alpha_{2}-\delta\right)\right]\left(\alpha_{2}-\delta\right) / \alpha_{2}^{2}\right)$.

3.2. Global Stability of $U_{3}^{(1)}=(\bar{u} ; \bar{v})$. Now let us define the conditions for which the stability of the product and the stock of capital of the economy is global; i.e., it does not depend on the produced quantities and level of stock at the initial moment. For this study, we define an appropriate Lyapunov function.

Theorem 6. Let us consider the following assumptions:

(3.1) admits a unique interior equilibrium point $U_{3}^{(1)}$

$$
=(\bar{u} ; \bar{v}),
$$

$$
0<\beta_{1}<4
$$

$\alpha_{2}>\delta$

$\beta_{2} d_{2}<2 \alpha_{2}$

$d_{2}<d_{1}$

$$
\begin{aligned}
& \frac{2 \delta \beta_{1}}{\alpha_{2}}<\frac{\alpha_{1}}{M_{u}+d_{2}} \\
& \frac{\beta_{1} M_{v}}{d_{2}}+\frac{\alpha_{1}}{d_{2}}<\frac{\beta_{1} d_{2}\left(2 \alpha_{2}-\delta\right)}{\alpha_{2}\left(M_{u}+d_{2}\right)}+\frac{\delta \beta_{1}\left(1+d_{2} \beta_{2} / 2 \alpha_{2}\right)}{\alpha_{2}}, \\
& \frac{1+\alpha_{1}}{d_{2}}-1+\frac{2 \beta_{1} d_{2}^{2}}{\alpha_{1}^{2}}<0
\end{aligned}
$$

where $M_{u}$ and $M_{v}$ are defined in Theorem 4.

If assumptions (25), (26), (27), (28), (29), (30), (31), (35), (36), and (37) are verified, then, the unique point of the interior equilibrium of model (3.1) is globally and asymptotically stable. 
Proof. Let us consider system (3.1). Let us suppose that assumption (35) is verified and, then, model (3.1) admits a unique interior equilibrium point $U_{3}^{(1)}=(\bar{u} ; \bar{v})$ verifying

$$
\begin{array}{r}
1=\bar{u}-\alpha_{1}\left(1-\frac{\bar{v}}{\bar{u}+d_{2}}\right), \\
\alpha_{2}-\delta=-\beta_{2} \bar{u}+\frac{\left(\beta_{2} \bar{u}+\alpha_{2}\right) \bar{v}}{\bar{u}+d_{2}} .
\end{array}
$$

Let us note that $\lambda=\beta_{1} d_{2} / 2 \alpha_{2}$. Given $V_{1}: \mathbb{R}^{2} \longrightarrow \mathbb{R}$ and $V_{2}: \mathbb{R}^{2} \longrightarrow \mathbb{R}$ one has

$$
\begin{aligned}
& V_{1}(u, v)=\left[u-\bar{u}-\bar{u} \ln \left(\frac{u}{\bar{u}}\right)\right]=\int_{\bar{u}}^{u}\left[1-\frac{\bar{u}}{x}\right] d x, \\
& V_{2}(u, v)=\lambda\left[v-\bar{v}-\bar{v} \ln \left(\frac{v}{\bar{v}}\right)\right]=\lambda \int_{\bar{v}}^{v}\left[1-\frac{\bar{v}}{x}\right] d x .
\end{aligned}
$$

Considering the Lyapunov functional $V: \mathbb{R}^{2} \longrightarrow \mathbb{R}$ one has

$$
V(u, v)=V_{1}(u, v)+V_{2}(u, v) .
$$

Let us pose

$$
\begin{aligned}
g(u, v)= & -1+\frac{\beta_{1} v^{2}+\alpha_{1} v}{\left(\bar{u}+d_{2}\right)\left(u+d_{2}\right)} ; \\
h(u, v)= & \frac{1}{2}\left[\beta_{1}-\frac{\beta_{1}(v+\bar{v})+\alpha_{1}}{\left(\bar{u}+d_{2}\right)}\right] \\
& +\frac{\lambda}{2}\left[\beta_{2}-\frac{\beta_{2} \bar{v}}{\bar{u}+d_{2}}+\frac{\left(\beta_{2} u+\alpha_{2}\right) v}{\left(\bar{u}+d_{2}\right)\left(u+d_{2}\right)}\right] .
\end{aligned}
$$

By using relation (29) and model (3.1), one obtains

$$
\frac{d V}{d t} \leq\left[\phi_{1}(u, v)\right](u-\bar{u})^{2}+\left[\phi_{2}(u, v)\right](v-\bar{v})^{2}
$$

with $\phi_{1}=g(u, v)+|h(u, v)|$ and $\phi_{2}=|h(u, v)|-\lambda\left(\beta_{2} u+\right.$ $\left.\alpha_{2}\right) /\left(\bar{u}+d_{2}\right), \forall(u, v) \in \mathbb{R}^{2}$.

Let us overestimate $g(u, v)$ and $h(u, v)$ and, then, $\phi_{1}(u, v)$ and $\phi_{2}(u, v)$.

(1) One has $g(u, v)<R_{0}<0, \forall(u, v) \in \mathbb{R}^{2}$, if $R_{0}=-1+$ $\left(\beta_{1} M_{v}^{2}+\alpha_{1} M_{v}\right) / d_{2}^{2}<0$.

(2) if $\beta_{2}<2 \alpha_{2} / d_{2}$ then $\partial h / \partial v<0$. So, $h\left(M_{u}, M_{v}\right) \leq$ $h(u, v) \leq h(0,0)$. Therefore, $h(0,0)<0$ if $\beta_{2} d_{2}<$ $2 \alpha_{2}, d_{2}<d_{1}, 2 \delta \beta_{1} / \alpha_{2}<\alpha_{1} /\left(M_{u}+d_{2}\right)$. Consequently, $|h(u, v)|=-h(u, v)<-h\left(M_{u}, M_{v}\right)<$ $R_{1}=\beta_{1} M_{v} / 2 d_{2}-\left(d_{2} \beta_{1} M_{v} / 2 \alpha_{2}\right)\left(\beta_{2} M_{u}+\alpha_{2}\right) /\left(M_{u}+\right.$ $\left.d_{2}\right)^{2}+(1 / 2)\left[-\left(\beta_{1} \delta / \alpha_{2}\right)\left(1+\beta_{2} d_{2} / 2 \alpha_{2}\right)+\alpha_{1} / d_{2}\right]$. Thus, $\phi_{1}(u, v)<R_{0}+R_{1}$ and $\phi_{2}(u, v)<R_{1}-\beta_{1} d_{2} / 2\left(M_{u}+d_{2}\right)$ if $\beta_{2} d_{2}<2 \alpha_{2}, d_{2}<d_{1}$, and $2 \delta \beta_{1} / \alpha_{2}<\alpha_{1} /\left(M_{u}+d_{2}\right)$.
Hence, $d V / d t<0, \forall(u, v) \in \mathbb{R}^{2}$, if

$$
\begin{aligned}
& 0<\beta_{1}<4, \\
& \alpha_{2}>\delta, \\
& \beta_{2}<\frac{\alpha_{2}}{d_{2}}, \\
& \frac{2 \delta \beta_{1}}{\alpha_{2}}<\frac{\alpha_{1}}{M_{u}+d_{2}}, \\
& \frac{\beta_{1} M_{v}}{2 d_{2}}+\frac{\alpha_{1}}{2 d_{2}} \\
& \quad<\frac{\beta_{1} d_{2}}{2\left(M_{u}+d_{2}\right)}+\frac{d_{2} \beta_{1} M_{v}}{2 \alpha_{2}} \frac{\left(\beta_{2} M_{u}+\alpha_{2}\right)}{\left(M_{u}+d_{2}\right)^{2}} \\
& \quad+\frac{\beta_{1} \delta}{2 \alpha_{2}}\left(1+\frac{\beta_{2} d_{2}}{2 \alpha_{2}}\right),
\end{aligned}
$$

and $\frac{\beta_{1} M_{v}^{2}+\alpha_{1} M_{v}}{d_{2}^{2}}-1+\frac{\beta_{1} d_{2}}{2\left(M_{u}+d_{2}\right)}<0$.

The model is permanent if $m^{u}=-\left(\beta_{1} / d_{2}\right) M_{v}^{2}-$ $\left(\alpha_{1} / d_{2}\right) M_{v}+1+\alpha_{1}>0$. Then, $\left(\beta_{1} / d_{2}^{2}\right) M_{v}^{2}+$ $\left(\alpha_{1} / d_{2}^{2}\right) M_{v}<\left(1+\alpha_{1}\right) / d_{2}$. Moreover, $1 /\left(M_{u}+d_{2}\right)<$ $4 d_{2} / \alpha_{1}^{2}$.

So, from formulas (35), one obtains (27) $\Longrightarrow(36)$ and $(28) \Longrightarrow(37)$.

Hence, $d V / d t<0, \forall(u, v) \in \mathbb{R}^{2}$, if assumptions (25)-(28) are verified.

Remark 7. As $\beta_{1}=0$, the unique interior equilibrium point of model (3.1) is globally and asymptotically stable if the following assumptions are verified:

$$
\begin{aligned}
\alpha_{2} & >\delta, \\
\beta_{2} & <\frac{\alpha_{2}}{d_{2}}, \\
\frac{\delta \beta_{2}}{\alpha_{2}} & <\frac{\alpha_{1}}{M_{u}+d_{2}}, \\
-1+\frac{1+\alpha_{1}}{d_{2}}+\frac{4 \alpha_{2} d_{2}}{\alpha_{1}^{2}} & <0, \\
\frac{\alpha_{1}}{2 d_{2}}+\frac{\delta \beta_{2}}{\alpha_{2}} & <\frac{3\left(\alpha_{2}-\delta\right)}{2\left(M_{u}+d_{2}\right)},
\end{aligned}
$$

with constant $M_{u}$ and $M_{v}$ defined in Theorem 4 .

In fact, it is worth taking the Lyapunov functional $V$ : $\mathbb{R}^{2} \longrightarrow \mathbb{R}$ so that $V(u, v)=V_{1}(u, v)+V_{2}(u, v)$ with $V_{1}: \mathbb{R}^{2} \longrightarrow$ $\mathbb{R}$ and $V_{2}: \mathbb{R}^{2} \longrightarrow \mathbb{R}$ so that

$$
\begin{aligned}
& V_{1}(u, v)=\left[u-\bar{u}-\bar{u} \ln \left(\frac{u}{\bar{u}}\right)\right]=\int_{\bar{u}}^{u}\left[1-\frac{\bar{u}}{x}\right] d x, \\
& V_{2}(u, v)=\left[v-\bar{v}-\bar{v} \ln \left(\frac{v}{\bar{v}}\right)\right]=\int_{\bar{v}}^{v}\left[1-\frac{\bar{v}}{x}\right] d x .
\end{aligned}
$$


Considering (38)-(40), one shows, similarly in $\beta_{1} \neq 0$, that $d V / d t<0, \forall(u, v) \in \mathbb{R}^{2}$.

\section{Border Dynamics of Plan $u w$}

On the plan $u w$ model (1.3) becomes

$$
\begin{aligned}
& \dot{u}(\tau)=\left[1+\alpha_{1}-u(\tau)\right] u(\tau), \\
& \dot{w}=-\lambda(w-u)-\lambda e^{-\lambda T} u(\tau-T), \\
& u, w \in C^{1}\left(\left[0 ;+\infty\left[; \mathbb{R}_{+}\right), u(0)>0, u_{0}=\varphi \in C\left([-T ; 0] ; \mathbb{R}_{+}\right)\right.\right. \\
& \text {with }\left(\alpha_{1}, T, \lambda\right) \in\left(\mathbb{R}_{+}^{*}\right)^{3} \text {. The equilibria of model }(4.1) \text { are } \\
& \qquad U_{0}^{(2)}=P_{u w}\left(E_{0}^{*}\right)=(0,0), \\
& U_{1}^{(2)}=P_{u w}\left(E_{1}^{*}\right)=\left(1+\alpha_{1} ; k\left(1+\alpha_{1}\right)\right) .
\end{aligned}
$$

Let $M_{u}^{(2)}=1+\alpha_{1}, 0<m_{u}^{(2)}<1+\alpha_{1}, M_{w}^{(2)}=k\left(1+\alpha_{1}\right)$, and $m_{w}^{(2)}=k m_{u}^{(2)}$. The model is permanent and the set defined by

$$
\begin{aligned}
\mathscr{A}^{(2)} & =\left\{(u, w) \in \mathbb{R}_{+}^{2}: m_{u}^{(2)} \leq u \leq M_{u}^{(2)}, \text { and } m_{w}^{(2)} \leq w\right. \\
\leq & \left.M_{w}^{(2)}\right\}
\end{aligned}
$$

is limited, positively invariant.

Concerning the local stability, one obtains, for any delay $T$, the following results:

(1) $U_{0}^{(2)}=(0 ; 0)$ is an unstable saddle point, repulsive along the direction $u$, and attractive along the direction $w$.

(2) $U_{1}^{(2)}=\left(1+\alpha_{1} ; k\left(1+\alpha_{1}\right)\right)$ is stable.

The global stability of equilibrium $U_{1}^{(2)}$ is given in the following theorem.

\section{Theorem 8.}

$$
\begin{aligned}
\text { If } T & <\frac{\ln 2}{\lambda} \\
\text { and } \lambda & <\left(1+\alpha_{1}\right)(1-\ln 2)
\end{aligned}
$$

and, then, the unique interior equilibrium point $U_{1}^{(2)}=(1+$ $\left.\alpha_{1} ; k\left(1+\alpha_{1}\right)\right)$ of model (4.1) is globally and asymptotically stable.

Proof. Let us consider $U_{1}^{(2)}=(\bar{u}, \bar{w})$ with $\bar{u}=1+\alpha_{1}$ and $\bar{w}=k\left(1+\alpha_{1}\right)$. Let us pose

$U=\ln (u / \bar{u})$ and $W=\ln (w / \bar{w})$. Then, one obtains the following system:

$$
\begin{aligned}
& \dot{U}(\tau)=-\bar{u}\left[e^{U(\tau)}-1\right], \\
& \dot{W}(\tau)=\frac{\lambda e^{-w(\tau)}}{k}\left[\left(e^{U(\tau)}-1\right)-k\left(e^{W(\tau)}-1\right)\right. \\
&\left.-e^{-\lambda T}\left(e^{U(\tau-T)}-1\right)\right] .
\end{aligned}
$$

Let us note that $V_{1}(\tau)=|U(\tau)|$.
Then, the superior derivative $D^{+} V_{1}(\tau)$ of $V_{1}(\tau)$ in relation to time, alongside the solutions of (46), gives

$$
D^{+} V_{1}(\tau) \leq-\bar{u}\left|e^{U(\tau)}-1\right|=-|u-\bar{u}| .
$$

Let us note that $V_{21}(\tau)=|W(\tau)|$. Considering $(u, w) \in \mathscr{A}^{(2)}$, one has $m_{u}^{(2)} / \bar{u} \leq e^{U(\tau)} \leq M_{u}^{(2)} / \bar{u}$ and $m_{w}^{(2)} / \bar{w} \leq e^{W(\tau)} \leq$ $M_{w}^{(2)} / \bar{w}$. Hence, the superior derivative of $V_{21}(\tau)$ in relation to time, alongside solutions (46), gives

$$
\begin{aligned}
D^{+} V_{21}(\tau) \leq & \lambda\left|e^{U(\tau)}-1\right|-\lambda \frac{w^{*}}{m_{w}^{(2)}}\left|e^{W(\tau)}-1\right| \\
& +\frac{M_{u}^{(2)} \lambda e^{-\lambda T}}{k} \int_{\tau-T}^{\tau}\left|e^{U(s)}-1\right| d s .
\end{aligned}
$$

Considering the functional: $V_{22}(\tau)=\left(M_{u}^{(2)} \lambda e^{-\lambda T} /\right.$ k) $\int_{\tau-T}^{\tau} \int_{v}^{\tau}\left|e^{U(s)}-1\right| d s d v$ then

$$
\begin{aligned}
D^{+} V_{22}(\tau) \leq & \frac{T M_{u}^{(2)} \lambda e^{-\lambda T}}{k}\left|e^{U(\tau)}-1\right| \\
& -\frac{M_{u}^{(2)} \lambda e^{-\lambda T}}{k} \int_{\tau-T}^{\tau}\left|e^{U(s)}-1\right| d s .
\end{aligned}
$$

Let us pose $V_{2}(\tau)=V_{21}(\tau)+V_{22}(\tau)$. Thus,

$$
\begin{aligned}
D^{+} V_{2}(\tau) \leq & \lambda\left(1+\frac{T M_{u}^{(2)} e^{-\lambda T}}{k}\right)\left|e^{U(\tau)}-1\right| \\
& -\lambda \frac{w^{*}}{m_{w}^{(2)}}\left|e^{W(\tau)}-1\right| .
\end{aligned}
$$

Then, $D^{+} V_{2}(\tau) \leq \frac{\lambda}{\bar{u}}\left(1+\frac{T M_{u}^{(2)} e^{-\lambda T}}{k}\right)|u-\bar{u}|$

$$
-\frac{\lambda}{m_{w}^{(2)}}|w-\bar{w}| .
$$

Let $V(\tau)=V_{1}(\tau)+V_{2}(\tau)$. Then,

$$
\begin{aligned}
D^{+} V(\tau) \leq & -\left[1-\frac{\lambda}{\bar{u}}\left(1+\frac{T M_{u}^{(2)} e^{-\lambda T}}{k}\right)\right]|u-\bar{u}| \\
& -\frac{\lambda}{m_{w}^{(2)}}|w-\bar{w}| .
\end{aligned}
$$

So, $D^{+} V(\tau)<0$ if $\left(1+\left(T e^{-\lambda T} /\left(1-e^{-\lambda T}\right)\right) M_{u}^{(2)}<\left(1+\alpha_{1}\right) / \lambda\right)$. Consequently, $D^{+} V(\tau)<0$ if $(T<(\ln 2) / \lambda$ and $\lambda<(1+$ $\left.\alpha_{1}\right)(1-\ln 2)$.

\section{Border Dynamics of the Plan $v w$}

On the $v w$ plan model (1.3) becomes

$$
\begin{aligned}
\dot{v}(\tau) & =\left[\alpha_{2}-\delta-\frac{\alpha_{2} v(\tau)}{d_{2}}\right] v(\tau), \\
\dot{w} & =-\lambda w-\lambda e^{-\lambda T} 1_{[0 ; T]}(\tau) u(\tau-T), \\
v, w & \in C^{1}\left(\left[0 ;+\infty\left[; \mathbb{R}_{+}\right) u(0)>0, u_{0}=\varphi \in C\left([-T ; 0] ; \mathbb{R}_{+}\right)\right.\right.
\end{aligned}
$$


with $\left(d_{2}, \alpha_{2}, \delta, T, \lambda\right) \in\left(\mathbb{R}_{+}^{*}\right)^{4}$ and $1_{[0 ; T]}(\tau)=1$ if $0 \leq \tau \leq$ $T, 1_{[0 ; T]}(\tau)=0$ if $\tau>T$.

The equilibria of model (5.1) are as follows: for $\tau \geq T$,

$$
\begin{aligned}
& U_{0}^{(3)}=P_{v w}\left(U_{0}\right)=(0,0), \\
& U_{2}^{(3)}=P_{v w}\left(U_{2}\right)=\left(\frac{\left(\alpha_{2}-\delta\right) d_{2}}{\alpha_{2}} ; 0\right) .
\end{aligned}
$$

Model (4.1) is nonpermanent. In fact, $\lim _{t \rightarrow+\infty}[w(t)]=0$.

Let us now give the results upon stability of the equilibria of model (5.1).

(1) The equilibrium $U_{0}^{(3)}=(0 ; 0)$ is stable if $\alpha_{2}<\delta$ and a point unstable saddle if $\alpha_{2}>\delta$.

(2) The equilibrium $U_{2}^{(3)}=\left(\left(\alpha_{2}-\delta\right) d_{2} / \alpha_{2} ; 0\right)$ for $\alpha_{2}>\delta$ is stable.

Moreover, the equilibrium $U_{2}^{(3)}=\left(\left(\alpha_{2}-\delta\right) d_{2} / \alpha_{2} ; 0\right)$ is globally and asymptotically stable. In effect, $\lim _{t \rightarrow+\infty}[v(t)]=\left(\alpha_{2}-\delta\right) d_{2} / \alpha_{2}$ and $\lim _{t \rightarrow+\infty}[w(t)]=$ 0 .

\section{Local Stability of Model (1.3)}

Let us consider model (1.3).

Let us pose that $U_{i}(\tau)=u(\tau)-u_{i}^{*}, V_{i}(\tau)=v(\tau)-v_{i}^{*}$, and $W_{i}(\tau)=w(\tau)-w_{i}^{*}$ for $i=0,1,2,3$ with

$$
\begin{aligned}
& u_{0}^{*}=0, \\
& u_{1}^{*}=1+\alpha_{1}, \\
& u_{2}^{*}=0, \\
& u_{3}^{*}=u^{*} \\
& v_{0}^{*}=0, \\
& v_{1}^{*}=0, \\
& v_{2}^{*}=\frac{\left(\alpha_{2}-\delta\right) d_{2}}{\alpha_{2}}, \\
& v_{3}^{*}=v^{*} \\
& w_{0}^{*}=0, \\
& w_{1}^{*}=k\left(1+\alpha_{1}\right), \\
& w_{2}^{*}=0, \\
& w_{3}^{*}=k u^{*} .
\end{aligned}
$$

The system linearized around the point of equilibrium $E_{i}^{*}=$ $\left(u_{i}^{*}, v_{i}^{*}, w_{i}^{*}\right), i=0,1,2,3$, is

$$
\begin{aligned}
& \dot{U}_{i}(\tau)=A_{11}^{(i)} U_{i}(\tau)+A_{12}^{(i)} V_{i}(\tau)+A_{13}^{(i)} W_{i}(\tau), \\
& \dot{V}_{i}(\tau)=A_{21}^{(i)} U_{i}(\tau)+A_{22}^{(i)} V_{i}(\tau), \\
& \dot{W}_{i}(\tau)=\lambda U_{i}(\tau)-\lambda W_{i}(\tau)-\lambda e^{-\lambda T} U_{i}(\tau-T),
\end{aligned}
$$

with

$$
\begin{aligned}
A_{11}^{(i)}= & 1-2 u_{i}^{*}+\left(\beta_{1} v_{i}^{*}+\alpha_{1}\right)\left(1-\frac{v_{i}^{*}}{u_{i}^{*}+d_{2}}\right) \\
& +\frac{\left(\beta_{1} v_{i}^{*}+\alpha_{1}\right) u_{i}^{*} v_{i}^{*}}{\left(u_{i}^{*}+d_{2}\right)^{2}}, \\
A_{12}^{(i)}= & {\left[\beta_{1}-\frac{2 \beta_{1}}{u_{i}^{*}+d_{2}} v_{i}^{*}-\frac{\alpha_{1}}{u_{i}^{*}+d_{2}}\right] u_{i}^{*}-\frac{\gamma w_{i}^{*}}{w_{i}^{*}+d_{1}}, } \\
A_{13}^{(i)}= & \frac{-\gamma d_{1} v_{i}^{*}}{\left(w_{i}^{*}+d_{1}\right)^{2}}, \\
A_{21}^{(i)}= & {\left[\beta_{2}\left(1-\frac{v_{i}^{*}}{u_{i}^{*}+d_{2}}\right)+\frac{\left(\beta_{2} u_{i}^{*}+\alpha_{2}\right) v_{i}^{*}}{\left(u_{i}^{*}+d_{2}\right)^{2}}\right] v_{i}^{*}, } \\
A_{22}^{(i)}= & \left(\beta_{2} u_{i}^{*}+\alpha_{2}\right)\left(1-\frac{2 v_{i}^{*}}{u_{i}^{*}+d_{2}}\right)-\delta, \\
A_{23}^{(i)}= & 0, \\
A_{31}^{(i)}= & \lambda, \\
A_{32}^{(i)}= & 0, \\
A_{33}^{(i)}= & -\lambda, \\
A_{34}^{(i)}= & -\lambda e^{-\lambda T .}
\end{aligned}
$$

The characteristic equation of (57) is

$$
\begin{aligned}
& \Delta_{i}(x, T)=P_{i}(x, T)+e^{-x T} Q_{i}(x, T)=0 \\
& \text { with } P_{i}(x, T)=-\left[x^{3}+a^{(i)} x^{2}+b^{(i)} x+c^{(i)}\right], \\
& Q_{i}(x, T)=e^{-\lambda T}\left[D^{(i)} x+E^{(i)}\right] \\
& \text { where, } D^{(i)}=-\lambda A_{13}^{(i)}, \\
& E^{(i)}=\lambda A_{13}^{(i)} A_{22}^{(i)} \\
& a^{(i)}=-\left(A_{11}^{(i)}+A_{22}^{(i)}-\lambda\right), \\
& b^{(i)}=-\left[\lambda\left(A_{11}^{(i)}+A_{22}^{(i)}+A_{13}^{(i)}\right)+A_{21}^{(i)} A_{12}^{(i)}-A_{11}^{(i)} A_{22}^{(i)}\right], \\
& c^{(i)}=\lambda\left[A_{11}^{(i)} A_{22}^{(i)}-A_{21}^{(i)} A_{12}^{(i)}+A_{13}^{(i)} A_{22}^{(i)}\right] .
\end{aligned}
$$

6.1. Local Stability of $E_{0}^{*}$ and $E_{1}^{*}$. We are in situations where $Q_{i}(x, T)=0$. Let us use the following criterion RouthHurwitz: the interior equilibrium $E_{i}^{*}$ is stable if $a^{(i)}>0$, $b^{(i)}>0, c^{(i)}>0$, and $a^{(i)} b^{(i)}-c^{(i)}>0$ and unstable if not.

Theorem 9. Let us suppose that $\alpha_{2}>\delta$. Then, the equilibria $E_{0}^{*}$ and $E_{1}^{*}$ of model (1.3) are all unstable.

Proof. Let us consider formula (63)-(67). 
(1) The equation of $E_{0}^{*}$ is $\Delta_{0}(x, T)=x^{3}+a^{(0)} x^{2}+b^{(0)} x+$ $c^{(0)}=0$.

One has the following: $a^{(0)}=\lambda-\left(1+\alpha_{1}+\alpha_{2}-\delta\right)$ and $b^{(0)}=\left(1+\alpha_{1}\right)\left(\alpha_{2}-\delta\right)-\lambda\left(1+\alpha_{1}+\alpha_{2}-\delta\right)$. Let us suppose that $\left(a^{(0)}>0\right.$ and $\left.b^{(0)}>0\right)$. Then, one would have $\left(1+\alpha_{1}+\alpha_{2}-\delta\right)<\lambda<\left(1+\alpha_{1}\right)\left(\alpha_{2}-\delta\right) /\left(1+\alpha_{1}+\alpha_{2}-\delta\right)$. Consequently, $\left(1+\alpha_{1}\right)^{2}+\left(\alpha_{2}-\delta\right)^{2}+\left(1+\alpha_{1}\right)\left(\alpha_{2}-\delta\right)<0$ which is absurd. Hence, the equilibrium $E_{0}^{*}$ of model (1.3) is unstable by application of the Routh-Hurwitz criterion.

(2) One has $\Delta_{1}(x, T)=x^{3}+a^{(1)} x^{2}+b^{(1)} x+c^{(1)}=0$. Meanwhile, $\alpha_{2}>\delta$ and, then, $c^{(1)}=-\lambda\left(1+\alpha_{1}\right)\left[\beta_{2}(1+\right.$ $\left.\left.\alpha_{1}\right)+\alpha_{2}-\delta\right]<0$ so the equilibrium $E_{0}^{*}$ of model (1.3) is unstable by application of Routh-Hurwitz criterion.

6.2. Local Stability of $E_{2}^{*}$ and $E_{3}^{*}$. We are in the situation where $Q_{i}(x, T) \neq 0$. In order to assess the influence of delay $T$ over the local stability of model (1.3), we use the results upon the local stability of model obtained taking $T=0$ into model (1.3) and the results of [5] upon the local stability of the delay systems. Let us consider the characteristic equation (63).

(i) One has the following: $Q_{i}(z, T)=0 \Longrightarrow z=$ $-E^{(i)} / D^{(i)} \in \mathbb{R}$. Then, $P_{i}(x, T)$ and $Q_{i}(x, T)$ do not have any common imaginary roots.

(ii) One has the following: $P_{i}(x, T)$ and $Q_{i}(x, T)$ are polynomials with real coefficients

so $\overline{P_{i}(-i \omega, T)}=P_{i}(i \omega, T)$ and $\overline{Q_{i}(-i \omega, T)}=Q_{i}(i \omega, T)$.

(iii) If $-(1 / \lambda) \ln \left(c^{(i)} / E^{(i)}\right) \neq T$ so $P_{i}(0, T)+Q_{i}(0, T)=$ $E^{(i)} e^{-\lambda T}-c^{(i)} \neq 0$.

(iv) Let us pose $x=\rho e^{i \varphi}$. Then, $\left|P_{i}(x, T) / Q_{i}(x, T)\right| \sim 1 / \rho^{4}$ when $\rho \longrightarrow+\infty$.

$$
\text { Thus } \limsup _{|x| \longrightarrow+\infty}\left\{\left|\frac{Q_{i}(x, T)}{P_{i}(x, T)}\right|\right\}=0<1 .
$$

Let us consider the function defined on $\mathbb{R}$ by the following: $F_{i}(\omega)=\left|P_{i}(i \omega, T)\right|^{2}-\left|Q_{i}(i \omega, T)\right|^{2}$.

$$
\begin{aligned}
\text { Then, } F(\omega)= & {\left[b^{(i)} \omega-\omega^{3}\right]^{2}+\left[c^{(i)}-a^{(i)} \omega^{2}\right]^{2} } \\
& -e^{-2 \lambda T}\left(D^{(i)} \omega^{2}+E^{(i)}\right) \\
\text { So } F_{i}(\omega)= & \omega^{6}+m_{2} \omega^{4}+m_{1} \omega^{2}+m_{0}, \text { where } \\
m_{2}= & \left(\left[a^{(i)}\right]^{2}-2 b^{(i)}\right), \\
m_{1}= & \left(\left[b^{(i)}\right]^{2}-2 a^{(i)} c^{(i)}-e^{-2 T \lambda} D^{(i)}\right), \\
m_{0}= & {\left[c^{(i)}\right]^{2}-E^{(i)} e^{-2 \lambda T} . }
\end{aligned}
$$

Let us determine $\delta\left(T_{i}^{*}\right)=\operatorname{sgn}\left(\left.(d \operatorname{Re}(x) / d T)\right|_{x=i \omega\left(T_{i}^{*}\right)}\right)$ the sign of the real part of a solution $x$ of the characteristic equation $\Delta_{i}(x, T)=0$.
Lemma 10. Let us consider $x$, a solution of the characteristic equation $\Delta_{i}(x, T)=P_{i}(x, T)+e^{-x T} Q_{i}(x, T)=0$, with

$$
\begin{aligned}
P_{i}(x, T) & =-\left[x^{3}+a^{(i)} x^{2}+b^{(i)} x+c^{(i)}\right] \\
\text { and } Q_{i}(x, T) & =e^{-\lambda T}\left\{D^{(i)} x+E^{(i)}\right\} .
\end{aligned}
$$

Given $\omega_{i}=\omega\left(T_{i}^{*}\right)$ positive root of $F_{i}(\omega)$ and $T_{i}^{*}$ the associated delay certifying the following relation:

$$
\begin{aligned}
T_{i}^{*} & =\frac{1}{\omega_{i}} \\
& \cdot \arctan \left\{\frac{\left(a^{(i)} D^{(i)}-E^{(i)}\right) \omega^{3}+\left(b^{(i)} E^{(i)}-c^{(i)} D^{(i)}\right) \omega}{-D^{(i)} \omega^{4}+\left(-a^{(i)} E^{(i)}+b^{(i)} D^{(i)}\right) \omega^{2}+c^{(i)} E^{(i)}}\right\} \\
& +\frac{2 n \pi}{\omega_{i}}, \quad n \in \mathbb{N} .
\end{aligned}
$$

Let us pose $\delta\left(T_{i}^{*}\right)=\operatorname{sgn}\left\{\left.(d \operatorname{Re}(x) / d T)\right|_{x=i \omega\left(T_{i}^{*}\right)}\right\}$. Then,

$$
\delta\left(T_{i}^{*}\right)=\operatorname{sgn}\left\{\Lambda_{1 i} H_{3 i}+\Lambda_{2 i} H_{1 i} H_{2 i}\right\}
$$

with

$$
\begin{aligned}
H_{1 i} & =\lambda^{2}+\omega^{2}, \\
H_{2 i} & =\left(\lambda E^{(i)}-D^{(i)} \omega^{2}\right)^{2}+\left(E^{(i)}+\lambda D^{(i)}\right)^{2} \omega^{2}, \\
H_{3 i} & =\left(c^{(i)}-a^{(i)} \omega^{2}\right)^{2}+\left(b^{(i)}-\omega\right)^{2} \omega^{2}, \\
\Lambda_{1 i} & =\left(-T_{i}^{*}\left[D^{(i)}\right]^{2}-\lambda^{2}\left[D^{(i)}\right]^{2}\right) \omega^{4}+\left\{\lambda D^{(i)} E^{(i)}\right. \\
& \left.-T_{i}^{*}\left[\left(E^{(i)}-\lambda D^{(i)}\right)^{2}-2 \lambda D^{(i)} E^{(i)}\right]-\lambda^{2}\left[D^{(i)}\right]^{2}\right\} \\
\cdot & \omega^{2}+\left(\lambda^{3} D^{(i)} E^{(i)}-\lambda^{2}\left[E^{(i)}\right]^{2} T_{i}^{*}\right), \\
\Lambda_{2 i} & =-3 a^{(i)} \omega^{4}+2 \omega^{3}+\left(3 c^{(i)}+a^{(i)} b^{(i)}-2 b^{(i)}\right) \omega^{2} \\
& -b^{(i)} c^{(i)} .
\end{aligned}
$$

Proof. Given $\omega_{i}=\omega\left(T_{i}^{*}\right)$ positive root of $F_{i}(\omega)$. Let us assume that there is a delay $T_{i}^{*}$ so that

$$
\begin{aligned}
T_{i}^{*} & =\frac{1}{\omega\left(T_{i}^{*}\right)} \arctan \left\{-\frac{\operatorname{Im}\left[P_{i}\left(i \omega, T_{i}^{*}\right) / Q_{i}\left(i \omega, T_{i}^{*}\right)\right]}{\operatorname{Re}\left[P_{i}\left(i \omega, T_{i}^{*}\right) / Q_{i}\left(i \omega, T_{i}^{*}\right)\right]}\right\} \\
& +\frac{2 n \pi}{\omega\left(T_{i}^{*}\right)}, \quad n \in \mathbb{N} .
\end{aligned}
$$

Then, $T_{i}^{*}=\frac{1}{\omega_{i}}$

$$
\begin{aligned}
& \cdot \arctan \left\{\frac{\left(a^{(i)} D^{(i)}-E^{(i)}\right) \omega^{3}+\left(b^{(i)} E^{(i)}-c^{(i)} D^{(i)}\right) \omega}{-D^{(i)} \omega^{4}+\left(-a^{(i)} E^{(i)}+b^{(i)} D^{(i)}\right) \omega^{2}+c^{(i)} E^{(i)}}\right\} \\
& +\frac{2 n \pi}{\omega_{i}}, \quad n \in \mathbb{N} .
\end{aligned}
$$


Let us pose

$$
\begin{aligned}
\delta\left(T_{i}^{*}\right) & =\operatorname{sgn}\left(\left.\frac{d \operatorname{Re}(x)}{d T}\right|_{x=i \omega\left(T_{i}^{*}\right)}\right) \\
& =\left[\operatorname{sgn}\left(\left.\frac{d \operatorname{Re}(x)}{d T}\right|_{x=i \omega\left(T_{i}^{*}\right)}\right)\right]^{-1} .
\end{aligned}
$$

One has

$$
\begin{aligned}
& \Delta_{i}(x, T)=P_{i}(x, T)+e^{-x T} Q_{i}(x, T)=0 \Longrightarrow \\
& \frac{d P_{i}(x, T)}{d T}-\left[T \frac{d(x)}{d T}+x\right] e^{-x T} Q_{i}(x, T) \\
& +e^{-x T} \frac{d Q_{i}(x, T)}{d T}=0 .
\end{aligned}
$$

Therefore, $P_{i}(x, T)=-\left[x^{3}+a^{(i)} x^{2}+b^{(i)} x+c^{(i)}\right]$ and $Q_{i}(x, T)=$ $e^{-\lambda T}\left\{D^{(i)} x+E^{(i)}\right\}$. Thus, $\left\{e^{-x T}\left[D^{(i)} e^{-\lambda T}-T Q_{i}(x, T)\right]-\left(3 x^{2}+\right.\right.$ $\left.\left.2 a^{(i)} x+b^{(i)}\right)\right\}(d(x) / d T)-e^{-x T}\left\{x Q_{i}(x, T)+\lambda e^{-\lambda T}\left(D^{(i)} x+\right.\right.$ $\left.\left.E^{(i)}\right)\right\}=0$.

$$
\begin{aligned}
& \text { Hence, }\left[\frac{d(x)}{d T}\right]^{-1} \\
& =\frac{\left\{e^{-x T}\left[D^{(i)} e^{-\lambda T}-T Q_{i}(x, T)\right]-\right.}{e^{-x T}\left\{x Q_{i}(x, T)+\lambda e^{-\lambda T}\right.} \\
& \text { Thus, }\left[\frac{d(x)}{d T}\right]^{-1} \\
& =-\frac{T}{x+\lambda}+\frac{D^{(i)}}{(x+\lambda)\left(D^{(i)} x+E^{(i)}\right)} \\
& -\frac{\left(3 x^{2}+2 a^{(i)} x+b^{(i)}\right)}{x^{3}+a^{(i)} x^{2}+b^{(i)} x+c^{(i)}}
\end{aligned}
$$$$
=\frac{\left\{e^{-x T}\left[D^{(i)} e^{-\lambda T}-T Q_{i}(x, T)\right]-\left(3 x^{2}+2 a^{(i)} x+b^{(i)}\right)\right\}}{e^{-x T}\left\{x Q_{i}(x, T)+\lambda e^{-\lambda T}\left(D^{(i)} x+E^{(i)}\right)\right\}} .
$$

and, meanwhile, $\delta\left(T_{i}^{*}\right)=\left[\operatorname{sgn}\left(\left.(d \operatorname{Re}(x) / d T)\right|_{x=i \omega\left(T_{i}^{*}\right)}\right)\right]^{-1}$. Then, $\delta\left(T_{i}^{*}\right)=\operatorname{sgn}\left\{\Lambda_{1 i} H_{3 i}+\Lambda_{2 i} H_{1 i} H_{2 i}\right\}$

with $H_{1 i}=\lambda^{2}+\omega^{2}, H_{2 i}=\left(\lambda E^{(i)}-D^{(i)} \omega^{2}\right)^{2}$ $+\left(E^{(i)}+\lambda D^{(i)}\right)^{2} \omega^{2}, H_{3 i}=\left(c^{(i)}-a^{(i)} \omega^{2}\right)^{2}+\left(b^{(i)}-\right.$ $\omega)^{2} \omega^{2}, \Lambda_{1 i}=\left(-T_{i}^{*}\left[D^{(i)}\right]^{2}-\lambda^{2}\left[D^{(i)}\right]^{2}\right) \omega^{4}+\left\{\lambda D^{(i)} E^{(i)}-\right.$ $\left.T_{i}^{*}\left[\left(E^{(i)}-\lambda D^{(i)}\right)^{2},-2 \lambda D^{(i)} E^{(i)}\right]-\lambda^{2}\left[D^{(i)}\right]^{2}\right\} \omega^{2}+\left(\lambda^{3} D^{(i)} E^{(i)}-\right.$ $\left.\lambda^{2}\left[E^{(i)}\right]^{2} T_{i}^{*}\right)$, and $\Lambda_{2 i}=-3 a^{(i)} \omega^{4}+2 \omega^{3}+\left(3 c^{(i)}+a^{(i)} b^{(i)}-\right.$ $\left.2 b^{(i)}\right) \omega^{2}-b^{(i)} c^{(i)}$.

It is noticed that the analysis of the local stability of the system with delay when $Q_{i}(x, T) \neq 0$ depends on the existence of positive root for function $F_{i}(\omega)$. The coefficients $m_{0}$ and $m_{1}$, of $F_{i}(\omega)$, depend on $T$. Therefore, its positive root $\omega$ depends on $T$. Using the Cartan method and Viet formulas, one proves on the one hand that $F_{i}(\omega)$ admits at least a positive root if $m_{0}<0$. On the other hand, if $m_{0} \geq 0$, then, $F_{i}(\omega)$ admits either of two positive roots whereas $F_{i}(\omega)$ does not admit any positive root.

The condition $m_{0}<0$ is equivalent to the following assumption:

$$
0 \leq T \leq T_{s}=-\frac{1}{2 \lambda} \ln \frac{\left[c^{(i)}\right]^{2}}{E^{(i)}}
$$

$$
\text { with } E^{(i)}>0 \text { and }\left[c^{(i)}\right]^{2}<E^{(i)} \text {. }
$$

Let us denote $\operatorname{det}\left[J^{(i)}\right]=A_{11}^{(i)} A_{22}^{(i)}-A_{21}^{(i)} A_{12}^{(i)}, p=-m_{1} m_{3}+$ $m_{2}^{2} / 3$, and $q=-m_{0} m_{3}^{2}-2\left(m_{2} / 3\right)^{3}+m_{1} m_{2} m_{3} / 3$. Then, one obtains the following proposition.

Proposition 11. Let us suppose that $A_{13}^{(i)} A_{22}^{(i)}>0$.

Let us pose $\lambda_{\max }^{(i)}=A_{13}^{(i)} A_{22}^{(i)} /\left[\operatorname{det}\left[J^{(i)}\right]+A_{13}^{(i)} A_{22}^{(i)}\right]^{2}$ and $T_{s}^{(i)}=-(1 / 2 \lambda) \ln \left(\left[c^{(i)}\right]^{2} / E^{(i)}\right), i=2,3$.

(1) $F_{i}(\omega)$ admits at least a positive root if

$$
\begin{aligned}
0 & \leq \lambda<\lambda_{\max }^{(i)} \\
\text { and } 0 & \leq T<T_{s}^{(i)} .
\end{aligned}
$$

(2) $F_{i}(\omega)$ does not admit any positive root if one of the following conditions is verified:

$$
\begin{aligned}
T_{s}^{(i)} & \leq T \\
\text { and } 27 q^{2}-4 p^{3} & >0 . \\
T_{s}^{(i)} & \leq T, \\
27 q^{2}-4 p^{3} & =0 \\
\text { and }\left[m_{1} m_{2}-m_{0}\right] & >0 . \\
T_{s}^{(i)} & \leq T, \\
27 q^{2}-4 p^{3} & <0, \\
m_{2} & <0, \\
m_{1} & <0 \\
\text { and }\left[m_{1} m_{2}-m_{0}\right] & >0 .
\end{aligned}
$$

Now, let us use the results of Theorem 4 from [5] to study the stability of these points of equilibriums $E_{2}^{*}$ and $E_{3}^{*}$.

Theorem 12. One poses

$$
x^{-}=\frac{\beta_{1} d_{2}+\alpha_{1}+\gamma d_{2} / d_{1}^{2}-\sqrt{\left(\beta_{1} d_{2}+\alpha_{1}+\gamma d_{2} / d_{1}^{2}\right)^{2}-4 \beta_{1} d_{2}\left(\gamma d_{2} / d_{1}^{2}-1\right)}}{2 \beta_{1} d_{2}} .
$$


Let us consider one of these assumptions (82)-(85) and one of the following conditions:

$$
\begin{aligned}
\frac{\gamma d_{2}}{d_{1}^{2}}<1, & \\
\frac{\gamma d_{2}}{d_{1}^{2}} \geq 1 & \\
\text { and } \max \left(0 ; x^{-}\right) & \leq \frac{\delta}{\alpha_{2}}<1, \\
\frac{\gamma d_{2}}{d_{1}^{2}} & \geq 1, \\
0 & \leq \frac{\delta}{\alpha_{2}}<\min \left(1 ; x^{-}\right) \\
\text {and } T & \neq-\frac{1}{\lambda} \ln \left(1-\frac{d_{1}^{2} \psi(\delta)}{\gamma \alpha_{2}\left(\alpha_{2}-\delta\right)}\right), \\
\operatorname{det}(J) & =A_{11}^{(3)} A_{22}^{(3)}-A_{21}^{(3)} A_{12}^{(3)}>0 \\
\text { and } \operatorname{Tr}(J) & =A_{11}^{(3)}+A_{22}^{(3)}<0 .
\end{aligned}
$$

With $\psi(\delta)$ defined in formula (23), consider the following.

(1) Let us suppose that one of assumptions (83)-(85) is verified for $i=2,3$. Then, there is no change of stability for equilibrium $E_{i}^{*}, i=2,3$.

(2) Let us suppose that assumptions (82) and one of these assumptions (87)-(89) are verified for $i=2$.

(a) If $\delta\left(T_{2}^{*}\right)>0$ then the equilibrium $E_{2}^{*}$ is unstable for any $T \in\left[0 ; T_{s}[\right.$.

(b) If $\delta\left(T_{2}^{*}\right)<0$ then the equilibrium $E_{2}^{*}$ is unstable if $0 \leq T<T_{2}^{*}$ and stable if $T_{2}^{*} \leq T$.

(3) Let us suppose that assumption (82) is verified for $i=3$.

(a) In case $\delta\left(T_{3}^{*}\right)>0$,

(i) If assumption (90) is verified then the equilibrium $E_{3}^{*}$ is stable if $0 \leq T<T_{3}^{*}$ and unstable if $T_{3}^{*} \leq T$.

(ii) If assumption (90) is not verified then the equilibrium $E_{3}^{*}$ is unstable for any $T \in$ $\left[0 ; T_{s}[\right.$.

(b) Considering $\delta\left(T_{3}^{*}\right)<0$,

(i) If assumption (90) is verified then the equilibrium $E_{3}^{*}$ is stable for every $T \in\left[0 ; T_{s}[\right.$.

(ii) If assumption (90) is not verified then the equilibrium $E_{3}^{*}$ is unstable if $0 \leq T<T_{3}^{*}$ and stable if $T_{3}^{*} \leq T$.

With $T_{i}^{*}$ and $\delta\left(T_{i}^{*}\right), i=2,3$ are the denotations defined in Lemma 10.

Proof. Let us consider formula (63)-(67). Let us consider the function $F_{i}(\omega)$ defined in relation to the formula (70)(71) for any point of equilibrium $E_{i}^{*}, i=2,3$. Let us consider assumptions (82). Then, $T_{s}^{(i)}$ exists so that, for any $T \leq T_{s}^{(i)}, F_{i}(\omega)$ admits $\omega_{i}=\omega\left(T_{i}^{*}\right)$ a positive root (see Proposition 11). Considering $T_{i}^{*}$ the associated delay determined from Lemma 10, consider the following:

(1) Let us suppose that one of assumptions (83)-(85) is verified in $\mathrm{i}=2,3$. Then, $\forall T>T_{s}^{(i)}, F_{i}(\omega)$ does not admit any positive root. Thus, there is no change of stability of the equilibrium $E_{i}^{*}, i=2,3$.

(2) Stability of $E_{2}^{*}$ : let us consider that one of assumptions (87)-(88) is verified. Then, $c^{(2)}<0$. If assumption (89) is verified, then, $T \neq T_{l}=-(1 / \lambda) \ln \left(c^{(2)} / E\right)$. Hence, $P_{2}(0, T)+Q_{2}(0, T)=E^{(2)} e^{-\lambda T}-c^{(2)} \neq 0$ if assumptions (87)-(88) are verified. Then, let us apply Theorem 4 from [5]. So, let us suppose that $0 \leq T<T_{s}^{(2)}$. It is known that $T=0$ and $E_{2}^{*}=U_{2}^{(1)}$ so $E_{2}^{*}$ is unstable because $\psi(\delta)>0$ for $\delta<\alpha_{2}$. Taking into account the conclusions of Theorem 4 from [5], one obtains the stability of $E_{2}^{*}$.

(3) Let us suppose that the assumption $\operatorname{det}(J)=A_{11}^{(3)} A_{22}^{(3)}-$ $A_{21}^{(3)} A_{12}^{(3)}>0$ of (90) is verified. Then, $c>B$ so $T \geq$ $0>T_{l}=-(1 / \lambda) \ln (c / B)$. Thus, let us apply Theorem 4 from [5]. It is known that when $T=0$ and $E_{3}^{(3)}=U_{3}^{(1)}$ then $E_{3}^{(3)}$ is stable if assumption (90) is verified and unstable if not. Taking into account the conclusions of Theorem 4 from [5], one gets the stability of $E_{3}^{*}$.

\section{Global Stability of Model (1.3)}

Theorem 13. Let us suppose that model (1.3) is permanent and that it admits a unique interior equilibrium point. If

$\delta<\alpha_{2}$

$\beta_{2} d_{2}-\alpha_{2}<0$

$d_{2}<d_{1}$

$\gamma<\alpha_{2}$

$T<\frac{\ln 2}{\lambda}$,

$\frac{1}{2}<\lambda$

$\frac{1+\alpha_{1}}{d_{2}^{2}}+\frac{\alpha_{2} M_{v}}{d_{2}^{2}}+\frac{4 d_{2}}{\alpha_{1}^{2}}\left(\frac{1+\alpha_{1}}{d_{1}}+\lambda\right)+\frac{\beta_{2} \delta}{\alpha_{2}}+\ln 2$

$-1<0$,

$\beta_{1} \frac{\delta}{\alpha_{2}}+\frac{\gamma}{M_{u}+d_{1}}<\frac{\alpha_{2}}{M_{u}+d_{2}}$

then the unique interior equilibrium point $E_{3}^{*}=$ $\left(u^{*} ; v^{*}, k u^{*}\right)$ of model $(1.3)$ is globally and asymptotically stable. 
Proof. Let us consider model (1.3) and $E_{3}^{*}=\left(u^{*} ; v^{*}, k u^{*}\right)$ a unique interior equilibrium point. Let us pose $U=\ln \left(u / u^{*}\right)$, $V=\ln \left(v / v^{*}\right)$, and $W=\ln \left(w / w^{*}\right)$.

Then, $u-u^{*}=u^{*}\left[e^{U}-1\right], v-v^{*}=v^{*}\left[e^{V}-1\right]$, and $w-w^{*}=w^{*}\left[e^{W}-1\right]$. Thus,

$$
\begin{aligned}
\dot{U} & =u^{*}\left[-1+\frac{\beta_{1} v^{2}+\alpha_{1} v}{\left(u^{*}+d_{2}\right)\left(u+d_{2}\right)}\right. \\
& \left.+\frac{\gamma k v}{\left(k u^{*}+d_{1}\right)\left(w+d_{1}\right) u}\right]\left\{e^{U}-1\right\}+v^{*}\left[\beta_{1}\right. \\
& \left.-\frac{\left(\beta_{1}\left(v+v^{*}\right)+\alpha_{1}\right)}{\left(u^{*}+d_{2}\right)}-\frac{\gamma k}{k u^{*}+d_{1}}\right]\left\{e^{V}-1\right\} \\
& +w^{*}\left[\frac{-d_{1}}{\left(k u^{*}+d_{1}\right) u}\right]\left\{e^{W}-1\right\}, \\
\dot{V} & =u^{*}\left[\beta_{2}-\frac{\beta_{2} v^{*}}{u^{*}+d_{2}}+\frac{\left(\beta_{2} u+\alpha_{2}\right) v}{\left(u^{*}+d_{2}\right)\left(u+d_{2}\right)}\right]\left\{e^{U}\right. \\
& -1\}-\frac{\left(\beta_{2} u+\alpha_{2}\right)}{u^{*}+d_{2}} v^{*}\left\{e^{V}-1\right\}, \\
\dot{W} & (\tau)=\frac{\lambda e^{-w(\tau)}}{k}\left[\left\{e^{U(\tau)}-1\right\}-k\left\{e^{W(\tau)}-1\right\}\right. \\
& \left.-e^{-\lambda T}\left\{e^{U(\tau-T)}-1\right\}\right] .
\end{aligned}
$$

Let us denote $V_{1}(\tau)=|U(\tau)|$. Consider

$$
\begin{aligned}
& (u, v, w) \in \mathscr{A}^{+}=\left\{(u, v, w) \in \mathbb{R}_{+}^{3}: m_{u}^{\varepsilon} \leq u \leq M_{u}, m_{v}\right. \\
& \left.\leq v \leq M_{v} \text { and } m_{w} \leq w \leq M_{w}\right\} .
\end{aligned}
$$

Then, $d V_{1}(\tau) / d \tau=\operatorname{sgn}(U(\tau)) \dot{U}(\tau)$. Meanwhile, $\left[\operatorname{sgn}\left(e^{U(\tau)}\right.\right.$ 1)] $\left(e^{U(\tau)}-1\right)=\left|e^{U(\tau)}-1\right|$ so the superior derivative of $V_{1}(\tau)$ in relation to the time, alongside the solutions of system (7.1), gives

$$
\begin{aligned}
D^{+} V_{1}(\tau) \leq & \left\{-1+\frac{\beta_{1} M_{v}^{2}+\alpha_{1} M_{v}}{d_{2}^{2}}+\frac{\gamma k M_{v}}{m_{u}^{\varepsilon} d_{1}^{2}}\right\}\left|u-u^{*}\right| \\
& +\left|\frac{\beta_{1} \delta}{\alpha_{2}}-\frac{\gamma k}{k M_{u}+d_{1}}\right|\left|v-v^{*}\right| \\
& +\frac{d_{1}}{\left(k M_{u}+d_{1}\right) M_{u}}\left|w-w^{*}\right| .
\end{aligned}
$$

Let us denote $V_{2}(\tau)=|V(\tau)|$. However, $\left(\beta_{2} u+\alpha_{2}\right) v /\left(u^{*}+\right.$ $\left.d_{2}\right)\left(u+d_{2}\right) \leq \alpha_{2} v /\left(u^{*}+d_{2}\right) d_{2}$ if $\beta_{2} d_{2}-\alpha_{2}<0$. Then,

$$
\begin{aligned}
D^{+} V_{2}(\tau) \leq & \left|\frac{\beta_{2} \delta}{\alpha_{2}}+\frac{\alpha_{2}}{d_{2}^{2}} M_{v}\right|\left|u-u^{*}\right| \\
& -\frac{\alpha_{2}}{M_{u}+d_{2}}\left|v-v^{*}\right| .
\end{aligned}
$$

Let us note $V_{31}(\tau)=|W(\tau)|$. One has $m_{u}^{\varepsilon} / u^{*} \leq e^{U(\tau)} \leq M_{u} / u^{*}$ and $m_{w} / w^{*} \leq e^{W(\tau)} \leq M_{w} / w^{*}$. Then, superior derivative of
$V_{31}(\tau)$ in relation to the time, alongside the solutions of (7.1), results in

$$
\begin{aligned}
D^{+} V_{31}(\tau) \leq & \lambda\left|e^{U(\tau)}-1\right|-\frac{\lambda}{m_{w}} w^{*}\left|e^{W(\tau)}-1\right| \\
& +\frac{M_{u} \lambda e^{-\lambda T}}{k} \int_{\tau-T}^{\tau}\left|e^{U(s)}-1\right| d s .
\end{aligned}
$$

Let us consider the functional $V_{32}(\tau)=\left(M_{u} \lambda e^{-\lambda T} /\right.$ k) $\int_{\tau-T}^{\tau} \int_{v}^{\tau}\left|e^{U(s)}-1\right| d s d v$; then,

$$
\begin{aligned}
D^{+} V_{32}(\tau) \leq & \frac{T M_{u} \lambda e^{-\lambda T}}{k}\left|e^{U(\tau)}-1\right| \\
& -\frac{M_{u} \lambda e^{-\lambda T}}{k} \int_{\tau-T}^{\tau}\left|e^{U(s)}-1\right| d s .
\end{aligned}
$$

Let us pose that $V_{3}(\tau)=V_{31}(\tau)+V_{32}(\tau)$. Thus,

$$
\begin{aligned}
D^{+} V_{3}(\tau) \leq & \lambda\left(1+\frac{T M_{u} e^{-\lambda T}}{k}\right)\left|e^{U(\tau)}-1\right| \\
& -\lambda\left|e^{W(\tau)}-1\right| .
\end{aligned}
$$

Then, $D^{+} V_{3}(\tau) \leq \frac{\lambda}{M_{u}}\left(1+\frac{T M_{u} e^{-\lambda T}}{k}\right)\left|u-u^{*}\right|$

$$
-\frac{2 \lambda}{m_{w}}\left|w-w^{*}\right| \text {. }
$$

Let us pose $V(\tau)=V_{1}(\tau)+V_{2}(\tau)+V_{3}(\tau)$. Then, from formulas (95), (96), and (100), one obtains

$$
\begin{aligned}
D^{+} V(\tau) \leq & h_{1}\left|u-u^{*}\right|+h_{2}\left|v-v^{*}\right| \\
& +h_{3}\left|w-w^{*}\right|
\end{aligned}
$$

$$
\text { if } \beta_{2} d_{2}-\alpha_{2}<0
$$

with $h_{1}=-1+\frac{\beta_{1} M_{v}^{2}+\alpha_{1} M_{v}}{d_{2}^{2}}+\frac{\gamma k M_{v}}{m_{u}^{\varepsilon} d_{1}^{2}}$

$$
\begin{gathered}
+\frac{\beta_{2} \delta}{\alpha_{2}}+\frac{\alpha_{2}}{d_{2}^{2}} M_{v} \\
+\frac{\lambda}{M_{u}}\left(1+\frac{T e^{-\lambda T}}{k} M_{u}\right), \\
h_{2}=-\frac{\alpha_{2}}{M_{u}+d_{2}}+\left|\frac{\beta_{1} \delta}{\alpha_{2}}-\frac{\gamma k}{k M_{u}+d_{1}}\right| \\
\text { and } h_{3}=-\frac{2 \lambda}{k m_{u}^{\varepsilon}}+\frac{d_{1}}{\left(k M_{u}+d_{1}\right) M_{u}} .
\end{gathered}
$$


We have: $T<\frac{\ln 2}{\lambda} \Longrightarrow$

$$
\begin{aligned}
\frac{e^{-\lambda T}}{k} & <1, \\
\beta_{1} \frac{\delta}{\alpha_{2}}+\frac{\gamma}{M_{u}+d_{1}} & <\frac{\alpha_{2}}{M_{u}+d_{2}} \Longrightarrow \\
h_{2} & <0, \\
\text { and } \frac{1}{2} & <\lambda \Longrightarrow \\
h_{3} & <0 .
\end{aligned}
$$

One has $\alpha_{1}^{2} / 4 d_{2}<M_{u} \Longrightarrow 1 / M_{u}<4 d_{2} / \alpha_{1}^{2}$. Model (1.3) is permanent. So, on the one hand, one has $m_{u}^{(0)} \geq m^{u}>0$ if $\left(\beta_{1} / d_{2}^{2}\right) M_{v}^{2}+\left(\alpha_{1} / d_{2}^{2}\right) M_{v}<\left(1+\alpha_{1}\right) / d_{2}^{2}$. In addition, $m_{u}^{(0)} / 2 \leq$ $m_{u}^{\varepsilon} \leq m_{u}^{(0)}$. Moreover, $m_{u}^{(0)} \geq 2 \sqrt{\gamma k M_{u} M_{v} / d_{1}}$.

Then, $\gamma k M_{v} / m_{u}^{\varepsilon} d_{1}^{2} \leq 2 \gamma M_{v} / m_{u}^{(0)} d_{1}^{2} \leq m_{u}^{(0)} / 2 k M_{u} d_{1}<$ $m_{u}^{(0)} / M_{u} d_{1}$ if $T<(\ln 2) / \lambda$.

So, $D^{+} V(\tau)<0$ if assumptions (91)-(93) are checked.

Corollary 14. For $r \geq 14$, if the following assumptions are verified:

$$
\begin{aligned}
\gamma & \leq 3+\left[1+2\left(r+\sqrt{r^{2}+r}\right)\right]^{2}, \\
d_{2} \geq & \frac{1-8 r \gamma-8 r+2 r \sqrt{(1 / 2 r-4 \gamma-4)^{2}-(8 / r)\left\{\gamma-3-\left[1+2\left(r+\sqrt{r^{2}+r}\right)\right]^{2}\right\}}}{8}, \\
\frac{d_{2}}{2 r} \geq & \alpha_{2} \geq \max \left\{\frac{4 d_{2}+4}{4 d_{2}-1} ; \frac{4 d_{2}+3+\left[1+2\left(r+\sqrt{r^{2}+r}\right)\right]^{2}}{4 d_{2}-1}\right\}+\gamma . \\
2\left(r+\sqrt{r^{2}+r}\right) & \leq \alpha_{1} \leq-1+\sqrt{\left(\alpha_{2}-\gamma\right)\left(4 d_{2}-1\right)-3-4 d_{2}} \\
0 & <\beta_{1}<\frac{1}{d_{2}}, \\
\beta_{2} & <\frac{\alpha_{2}}{d_{2}}, \\
\frac{1}{2} & <\lambda<1+\alpha_{1}, \\
\beta_{2} & <\beta_{2}^{\text {max }}=\frac{\alpha_{2}}{M_{u}}\left[\frac{d_{2}}{M_{u}+d_{2}}+\frac{\delta}{\alpha_{2}}-1\right] \\
d_{2} & <d_{1}, \\
\frac{d_{2}}{M_{u}+d_{2}} & <\frac{\delta}{\alpha_{2}}<1, \\
&
\end{aligned}
$$

then, the unique interior equilibrium point $E_{3}^{*}=\left(u^{*} ; v^{*}, k u^{*}\right)$ of model (1.3) is globally and asymptotically stable.

Proof. Let us consider the assumptions of Theorem 13.

$$
\text { Posing: } \chi\left(\beta_{1}\right)= \begin{cases}d_{2} & \text { si } 0<\beta_{1}<\frac{1}{d_{2}} \\ \sqrt{\frac{d_{2}}{\beta_{1}}} & \text { si } \frac{1}{d_{2}}<\beta_{1}<4 .\end{cases}
$$

The model is permanent. Then

$$
\begin{aligned}
& m^{(0)} \geq m^{u}>0 \Longleftrightarrow \\
& \left(1-\frac{\chi\left(\beta_{1}\right)}{M_{u}+d_{2}}<\frac{\delta}{\alpha_{2}}<1, \beta_{2}<\beta_{2}^{\text {max }}\right. \\
& \left.=\frac{\alpha_{2}}{M_{u}}\left[\frac{\chi\left(\beta_{1}\right)}{M_{u}+d_{2}}+\frac{\delta}{\alpha_{2}}-1\right]\right) .
\end{aligned}
$$


Assumptions (91) and (93) give the following assumption:

$$
\beta_{1}<\frac{\alpha_{2}-\gamma}{M_{u}+d_{2}}
$$

Moreover, assumptions (91) and (92) give the following assumption:

$$
\frac{1+\alpha_{1}}{d_{2}^{2}}+\frac{2 \alpha_{2}}{d_{2}}+\frac{4 d_{2}}{\alpha_{1}^{2}}\left(\frac{1+\alpha_{1}}{d_{1}}+\lambda\right)+\ln 2-1<0
$$

Posing $\left(1+\alpha_{1}\right) / d_{2}^{2}=1 / r_{1}, 2 \alpha_{2} / d_{2}=1 / r_{2},\left(4 d_{2} / \alpha_{1}^{2}\right)((1+$ $\left.\left.\alpha_{1}\right) / d_{1}\right)=1 / r_{3}$, and $4 d_{2} \lambda / \alpha_{1}^{2}=1 / r_{4}$.

Then, condition (113) becomes

$$
\frac{1}{r_{1}}+\frac{1}{r_{2}}+\frac{1}{r_{3}}+\frac{1}{r_{4}}<1-\ln 2
$$

By imposing the condition, $r_{1}=r_{2}=r_{3}=r_{4}=r$, one obtains $r \geq 14$ and assumptions (103)-(109). According to Theorem 13, the unique interior equilibrium point $E_{3}^{*}=$ $\left(u^{*} ; v^{*}, k u^{*}\right)$ of model $(1.3)$ is globally and asymptotically stable.

\section{Numerical Simulations}

8.1. Limit Cycle. Let us consider the period $p=46$ and the following control parameters: $\left(d_{1}, d_{2}, \gamma, \alpha_{1}, \alpha_{2}, \delta, \beta_{1}, \beta_{2}\right)=$ $(1.75 ; 0.25 ; 0.5 ; 3.5 ; 1.75 ; 0.4375 ; 3.617 ; 1.1), \lambda=0.0000123$, and $T=45$.

For the graphic illustration, let us consider the initial conditions $\left(u_{0}, v_{0}, w_{0}\right)=(6 ; 3 ; 0.00331962170895946)$, the step $h=10^{-5}$, and the numbers iterations of time $N=$ 4600000. One obtains the maximum values of $u, v$, and $w$ :

$$
\begin{aligned}
& u_{\text {max }}=66.4527115210953, \\
& v_{\text {max }}=2836.21769136928, \\
& w_{\text {max }}=0.0367713984036395 .
\end{aligned}
$$

The trivial equilibrium points of the model are $E_{0}=$ $(0 ; 0.0)$,

$E_{1}=(4.5 ; 0 ; 0.00249006081209868)$, and $E_{2}=$ $(0 ; 0.000476921218349683 ; 0)$.

In addition, $E^{*}=(2.19899664564055 ; 2.19198952607149$; 0.00121680786072133 ) is the unique interior equilibrium. So, the illustrating Figure 1 is for the initial time $t_{0}=0$.

(i) Interpretation. The trajectories of GDP $u$ and of capital $v$ and $w$ (the effect of delay) are periodic. The orbit starting from the initial condition $\left(u_{0}, v_{0}, w_{0}\right)$ gravitates around the equilibrium point $E^{*}$ without never reaching it. There is, therefore, a limit cycle around this equilibrium point $E^{*}$. The orbit revolves around the equilibrium point. They are moving away from trivial equilibrium points and converge towards the limit cycle around $E^{*}$. Indeed, one has $\lambda=0.0000123<$ $\lambda_{\max }^{(3)}=0.2302$ and $T=45<T_{s}^{(3)}=305733.267595647$. According to Proposition 11, $\omega=0.000686044679105366$ is the positive root of $F(\omega)$. One has $T_{3}^{*}=2383.38276771532$ and $\delta\left(T_{3}^{*}\right)<0$. So, $E^{*}$ is unstable according to Theorem 12 because $\operatorname{Tr}=3.255>0$ for $T=0$. The trivial equilibrium points of the model are all unstable for $T<T_{3}^{*}<T_{2}^{*}=$ 4437.41337922542. Hence, we have the graphic illustration.

8.2. Global Stability. Let us consider the period $p=25$ and the following control parameters: $\left(d_{1}, d_{2}, \gamma, \alpha_{1}, \alpha_{2}, \delta, \beta_{1}, \beta_{2}\right)$ $=(101390.241 ; 50695.121 ; 0.5 ; 1822.498 ; 1747.022 ; 1721.154$; $9.863 .10^{-6} ; 7.784 .10^{-6}$ ) and $\lambda=0.75$ and $T=0.462$.

For the graphic illustration, let us consider the initial conditions $\left(u_{0}, v_{0}, w_{0}\right)=(5000 ; 8000 ; 1344.89438670147)$, the step $h=10^{-4}$, and the numbers iterations of time $N=$ 250000. From condition (9) of Theorem 4, the system is permanent if $\alpha_{2}>\delta, 0 \leq \beta_{1}<4$, and $m_{u}^{(0)} \geq 2 \sqrt{\gamma k M_{u} M_{v} / d_{1}}$. One obtains the maximum and minimum values of $u, v$, and $w$ for $\varepsilon=m_{u}^{(0)}-2 \sqrt{\gamma k M_{u} M_{v} / d_{1}}$ :

$$
\begin{aligned}
& u_{\text {max }}=1661666.077, \\
& v_{\text {max }}=38031.766, \\
& w_{\text {max }}=486690.726 . \\
& u_{\text {min }}=m_{u}^{\varepsilon}=1139.233, \\
& v_{\text {min }}=750.460, \\
& w_{\text {min }}=333.6737 .
\end{aligned}
$$

The trivial equilibrium points of the model are $E_{0}=(0 ; 0 ; 0)$, $E_{1}=(1823.498 ; 0 ; 534.090)$, and $E_{2}=(0 ; 750.630 ; 0)$.

In addition, $E^{*}=(1796.505 ; 777.6445 ; 526.184)$ is the unique interior equilibrium point verifying condition (91)-(93) of Theorem 13 . Then, $E^{*}$ is globally stable. Hence, the illustrating Figure 2 is for the initial time $t_{0}=0$ :

(ii) Interpretation. The trajectories of GDP $u$ and capital $v$ and $w$ (the effect of delay) stabilize, respectively, around $u^{*}=1796.505, v^{*}=777.6445$, and $w^{*}=526.184$ when $t$ is greater than $T$. The border dynamics show that when the orbit arrives in a border plan, it remains and converges towards a corresponding equilibrium point (see Orbit. 2 to Orbit. 4 of Figure 2). Moreover, the trivial equilibrium points of the model are all unstable. So, the orbits, are moving away from the borders plans and converge towards $E^{*}$. Hence, $E^{*}$ is globally stable, which is illustrated by the figure "phase portrait" of Figure 2.

\section{Conclusion}

The economic basic model of our work was one of dynamics Kaldor at an effective growth rate whose rate of saving is the Holling-II type and investment rate is from Leslie and Gower type modified in dimension two. Taking into account the time required for the saving to ensure its self-financing of all the investments, one obtains a model with a delay of the Kaldor type modified. This model is bounded and 

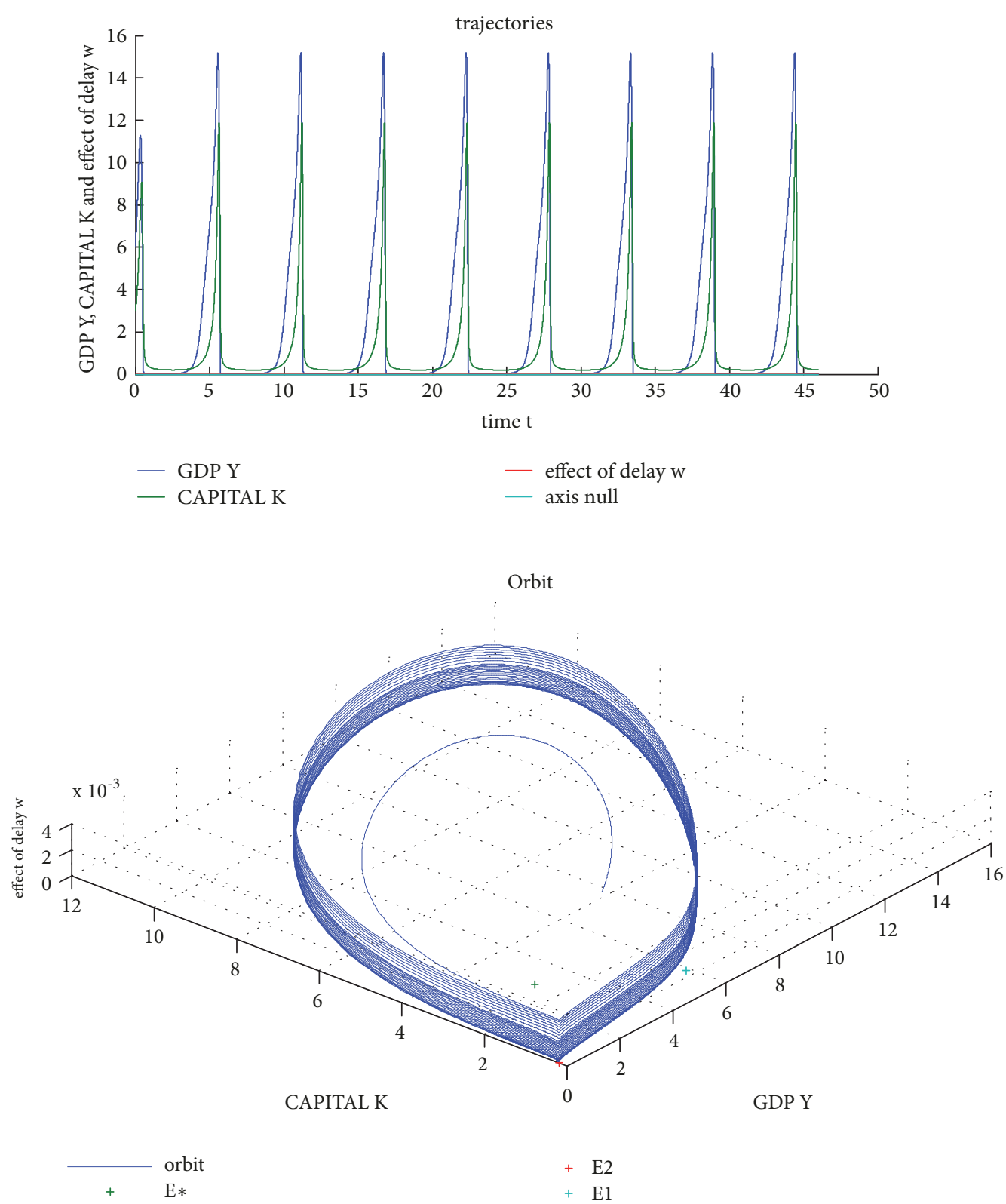

FIGURE 1: Trajectories and orbits with $u_{0}(\theta)=\varphi(\theta)=u(0) e^{A \theta} /\left(1+u(0)\left(e^{A \theta}-1\right)\right), \forall \theta[-T ; 0]$, where $A=(1 / 2 T) \ln \{|u(0)-1| / u(0)\}$ if $u(0) \neq 1$ and $A=-1 / 2 T$ if $u(0)=1$.

admits an attractor unit (set). Thus, under certain conditions, this model with delay is permanent. On the one hand, this permanence appears in the form of stationary growth of the capital stock and the product (stable interior equilibrium point) and, on the other hand, in the form of cyclic growth of the capital and the product (limiting cycle). In other words, this permanence avoids a shortage of the capital stock or the long-term production. In front of some other conditions, the dynamic stability with delay is also global; i.e., it does not depend on the level of the capital stock and the level of the production at the initial time. The consideration of the delay (due to the time for the economy to finance investments) can justify the bifurcation of an economic model of the stationary growth towards cyclic growth (see Theorem 12, (3)(a)(i)). However, this delay in the model can stabilize an initially unstable equilibrium of the system (see Theorem 12, (3)(b)(ii)), which represents a major economic interest since it allows the saving to get rid of risks from the cyclic growth.

\section{Data Availability}

No data were used to support this study.

\section{Conflicts of Interest}

The authors declare that there are no conflicts of interest for this paper. 

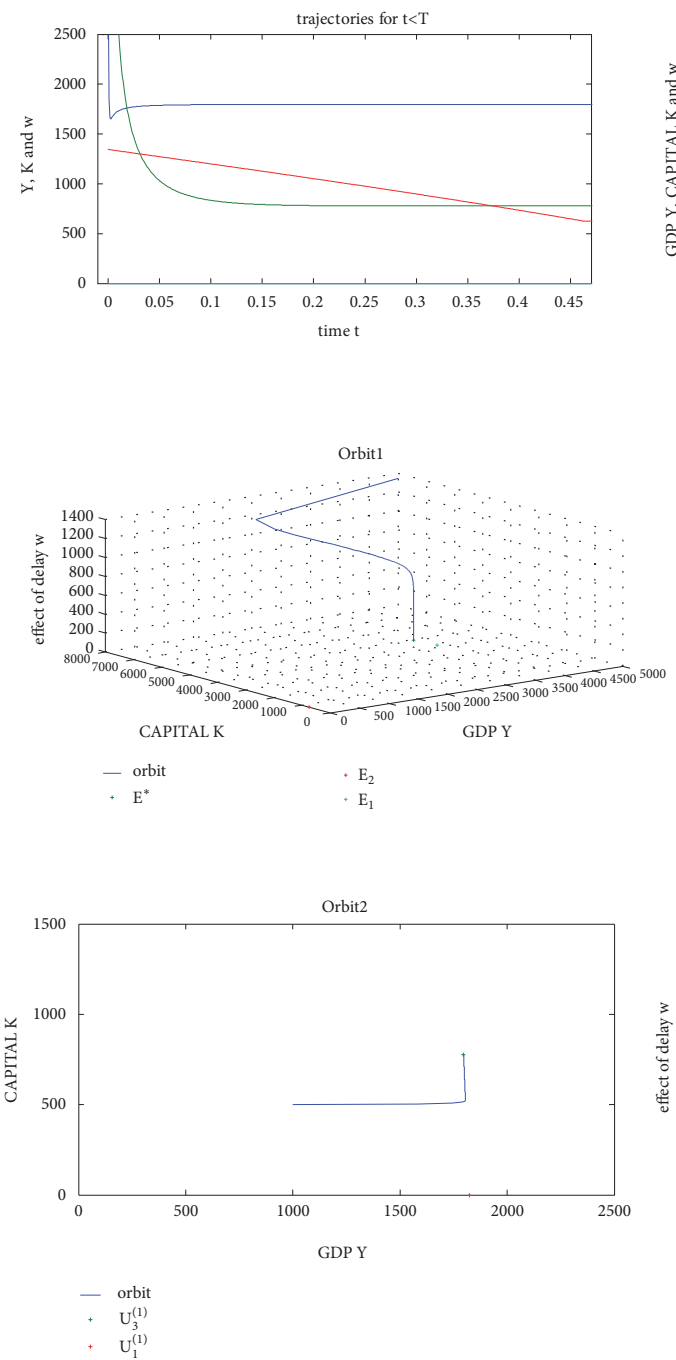
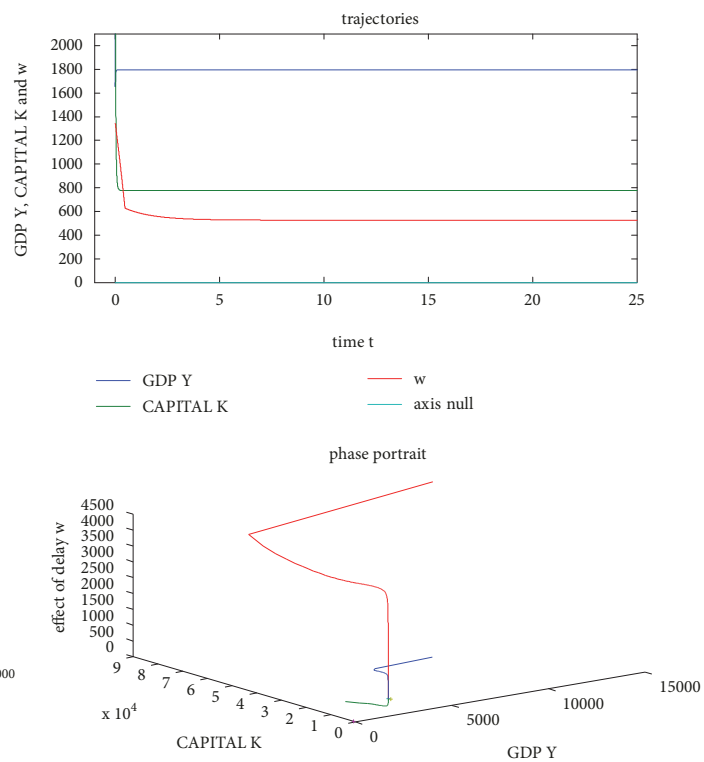

$\begin{array}{ll}- \text { orbit01 } & +E^{*} \\ - \text { orbit02 } & +E_{2} \\ - \text { orbit03 } & +E_{1}\end{array}$

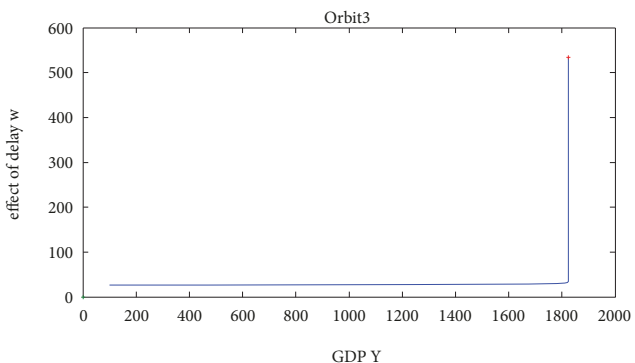

$\begin{array}{cc}- & \text { orbit } \\ + & \mathrm{U}_{0}^{(2)} \\ + & \mathrm{U}_{1}^{(2)}\end{array}$

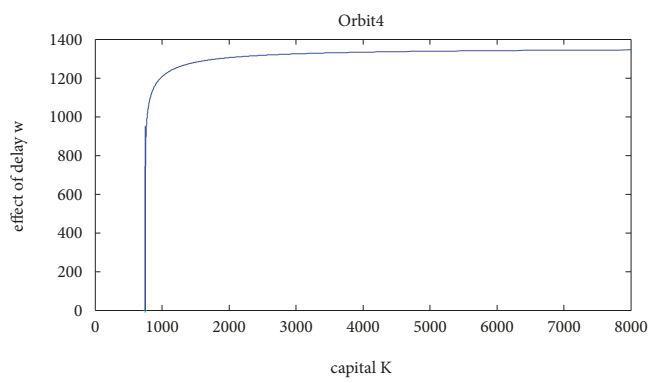

$\begin{array}{ll}- & \text { orbit } \\ + & \mathrm{U}_{2}^{(3)}\end{array}$

FIgURE 2: Trajectories, orbits, and phase portrait with $u_{0}(\theta)=\varphi(\theta)=u(0) e^{A \theta} /\left(1+u(0)\left(e^{A \theta}-1\right)\right), \forall \theta[-T ; 0]$, where $A=(1 / 2 T) \ln \{\mid u(0)-$ $1 \mid / u(0)\}$ if $u(0) \neq 1$ and $A=-1 / 2 T$ if $u(0)=1$.

\section{References}

[1] A. F. Nindjin, A. Tetchi N’Guessan, H. Okou, and K. Thiban Tia, "Cyclic growth and global stability of economic dynamics of Kaldor type in two dimensions," International Journal of Differential Equations, Art. ID 2062819, 12 pages, 2017.

[2] A. F. Nindjin, A. T. N'Guessan, H. Okou, and K. T. Tia, "Bifurcations of an economic dynamics of a modified Kaldor type in two dimensions," Global Journal of Pure and Applied Mathematics, vol. 2017, pp. 7855-7877, 2017, http://www.ripublication.com/gjpam.htm.

[3] H.-W. Lorenz, Nonlinear Dynamical Economics and Chaotic Motion, Springer, Berlin, Germany, 1993.

[4] F. D. Chen, X. Y. Liao, and Z. K. Huang, "The dynamic behavior of $\mathrm{N}$-species co-operation system with continuous time delays and feedback controls," Applied Mathematics and Computation, vol. 181, no. 2, pp. 803-815, 2006. 
[5] E. Beretta and Y. Kuang, "Geometric stability switch criteria in delay differential systems with delay dependent parameters," SIAM Journal on Mathematical Analysis, vol. 33, no. 5, pp. 11441165, 2002. 


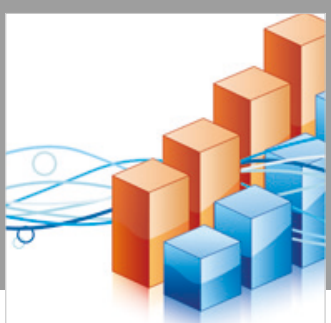

Advances in

Operations Research

\section{-n-m}
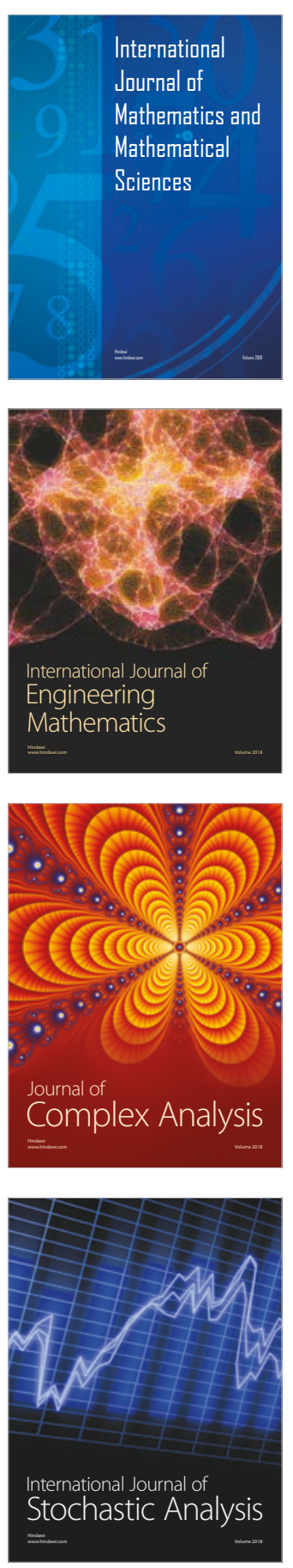
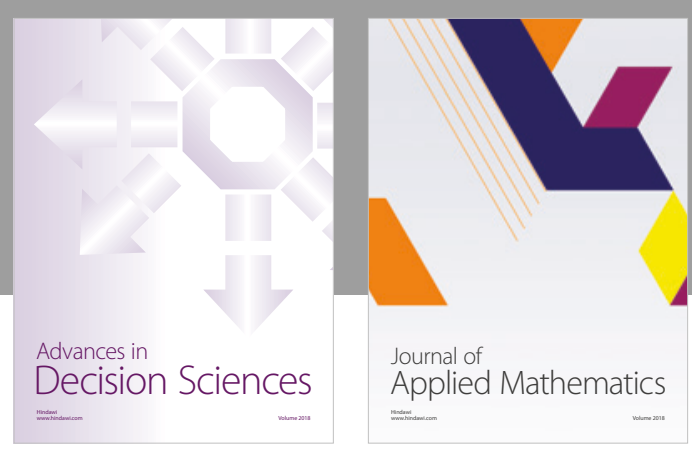

Journal of

Applied Mathematics
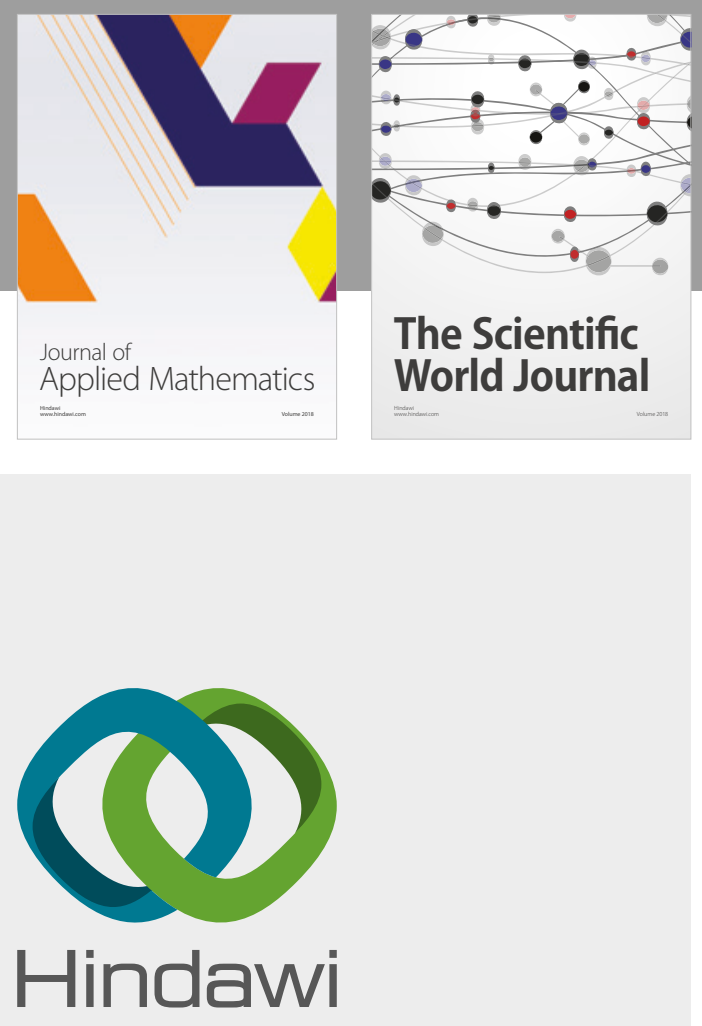

Submit your manuscripts at

www.hindawi.com

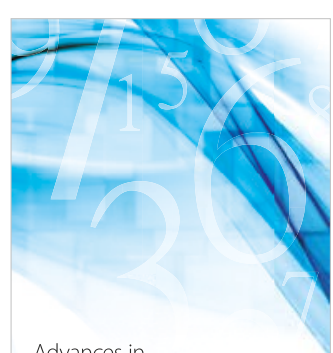

Advances in
Numerical Analysis
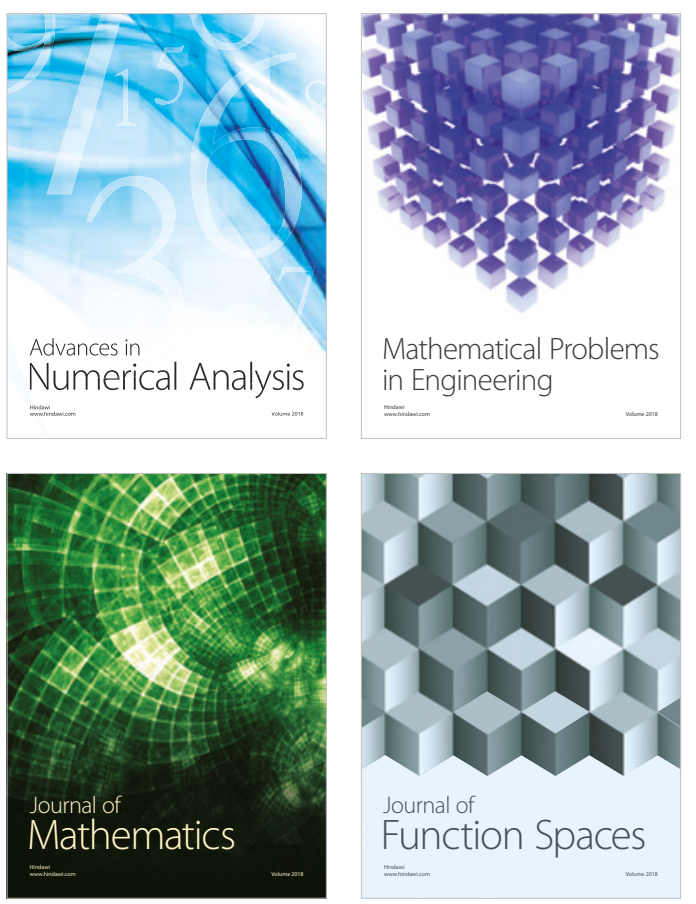

Mathematical Problems in Engineering

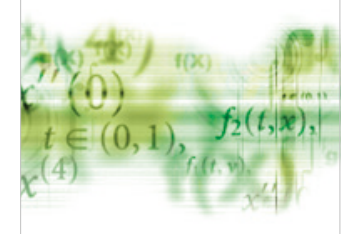

International Journal of

Differential Equations

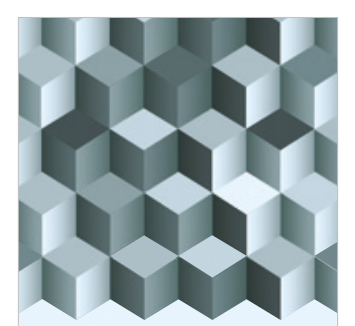

Journal of

Function Spaces

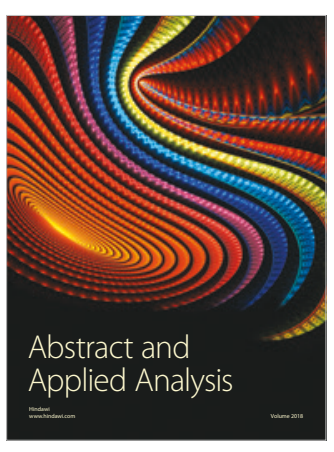

The Scientific

World Journal

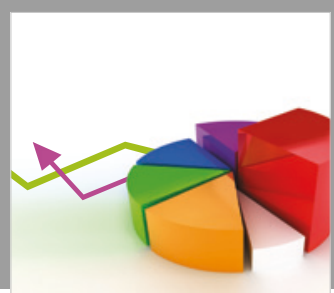

Journal of

Probability and Statistics
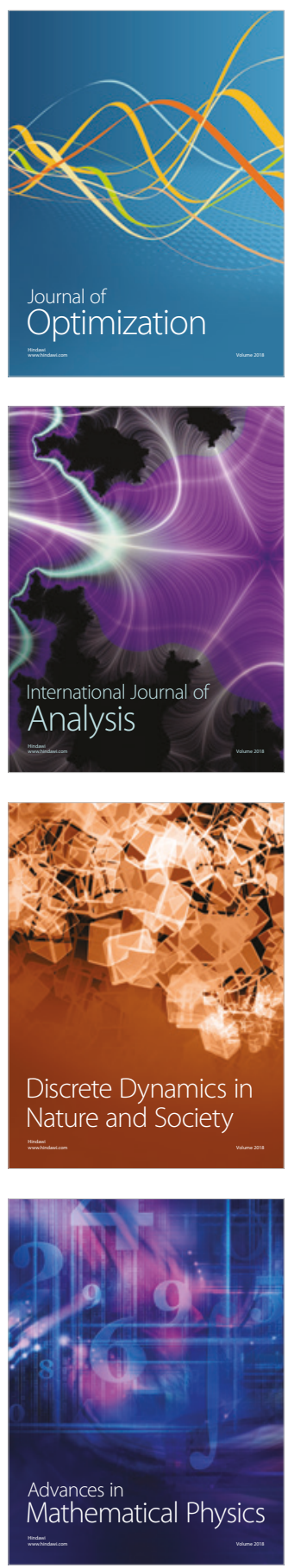